\title{
Effects of radiation and triaxiality of triangular equilibrium points in elliptical restricted three body problem
}

\author{
A Narayan ${ }^{1 *}$, Krishna Kumar Pandey ${ }^{2}$, Sandip Kumar Shrivastava ${ }^{2}$ \\ ${ }^{1}$ Department of Mathematics, Bhilai Institute of Technology, Durg, 491001, India \\ ${ }^{2}$ Department of Mathematics, ShriShankaracharya Institute of Technology and Management,Bhilai,490020,India \\ *Corresponding author E-mail:ashutoshmaths.narayan@gmail.com
}

\begin{abstract}
This paper studies effects of the triaxiality and radiation pressure of both the primaries on the stability of the infinitesimal motion about triangular equilibrium points in the elliptical restricted three body problem(ER3BP), assuming that the bigger and the smaller primaries are triaxial and the source of radiation as well. It is observed that the motion around these points is stable under certain condition with respect to the radiation pressure and oblate triaxiality. The critical mass ratio depends on the radiation pressure, triaxiality, semi -major axis and eccentricity of the orbits. It is further analyzed that an increase in any of these parameters has destabilizing effects on the orbits of the infinitesimal.
\end{abstract}

Keywords:ER3BP; Dynamical System ,Libration Points; Stablity

\section{Introduction}

The present paper is devoted to the analysis of the effects of the radiation and triaxiality of both the primaries on the stability of infinitesimal moving around the triangular equilibrium points of the elliptical restricted three-body problem. The elliptical restricted three-body problem is the generalization of the classical restricted three-body problem, it possesses some of the useful property of circular restricted three-body problem. This model has a restriction that the third body(infinitesimal mass) has no effects on the two massive bodies(Primaries). The eccentricity of the orbits plays a significant role which might not be seen in the circular case .The orbits of most celestial bodies are elliptical rather than circular as such; the elliptical restricted three-body problem describes the dynamical system more accurately. In elliptical restricted three body problems, the primaries move in elliptical orbits about their common centre of mass, without being affected by the third body of infinitesimal mass. The position of the primaries is fixed and the coordinates are obtained by dividing them by variable distance between the primaries. The Hamiltonian of the system depends explicitly on time. The bodies of the elliptical restricted three body problems are generally considered to be spherical in shape, but in actual situations, it is observed that several heavenly bodies are oblate spheroid or triaxial rigid body. The planet Earth, Jupitar and Saturn as well as stars Archid, Archerner, Antares, Altairand and Luyten areeither sufficiently oblate or triaxial rigid bodies and are significant in study of celestial bodies and stellar system. The lacks of spherity of heavenly bodies causes a large perturbation.

The elliptical restricted three-body problem has been described in detail by Danby(1964), Bennet(1965), Szebehely(1967), Markeev(1978).The The problem was further generalised by taking certain specific characteristics of celestial bodies like oblateness and triaxiality. The influence of eccentricity of orbits of the prima- ries with or without radiation pressure(s) and the triaxiality of the primaries on the existence and stability of the equilibrium points were studied by Gyorgyey(1985), Kumar Kumar and Choudhary (1990),Markellos et.al. (1992), Zimvoschikov and Thakai(2004), Ammar(2008), Narayan and Kumar(2011), Singh and Aishetu (2012 a), Singh and Aishetu (2012 b), Kumar and Ishwar(2014), Narayan and Usha(2014),Usha et al.(2014), and El-Salam(2015).

The present study is an attempt which is devoted to the analysis of the stability of triangular points, assuming that the bigger and smaller primaries are oblate triaxial and the source of radiation as well. This study will contribute to understand the effects of oblateness, radiation, eccentricity and triaxiality on the celestial dynamical systems.

This paper has five sections, section-1 describes introduction, section-2 provides the equation of motion, while section-3 describes the location of the triangular equilibrium points; similarly section- 4 contains stability of the equilibrium points and in the last section-5, discussion and conclusion are drawn.

\section{Equation of motion}

Suppose there are two bodies $S_{1}$ and $S_{2}$ of masses $m_{1}$ and $m_{2}$ with $\mathrm{m}_{1} \geq \mathrm{m}_{2}$ moving in a plane about their common centre of mass $\mathrm{O}$ in Keplerian elliptical orbit having eccentricity e. It is further assumed that the bigger and the smaller primariesm $m_{1}$ and $\mathrm{m}_{2}$ oblate triaxial and are the source of radiation as well. A third body $P$ of infinitesimal mass moves in the plane of motion $S_{1}$ and $S_{2}$ under their gravitational attraction but without affecting their motion. The motion of the smaller primary is not affected by the bigger primary, but the motion of both the primaries affects the motion of the infinitesimal mass. Consider the equation of motion in a fixed co-ordinate system using dimensional quantities and variables The dimensionless variables are introduced by using the distance $r$ between the primaries given by: 


$$
r=\frac{a\left(1-e^{2}\right)}{1+e \cos v}
$$

where a and e are Semi-major axis and the eccentricity of the elliptical orbit of the either primary around the other and $v$ is the true anomaly. Suppose $\mathrm{P}(\mathrm{x}, \mathrm{y})$ are the co-ordinate of the infinitesimal mass of the primaries and the line joining the primaries is taken as $\mathrm{X}$-axis. The $\mathrm{Y}$-axis is taken as the line passing through $\mathrm{O}$ and perpendicular to the $\mathrm{X}$-axis. Let $\mathrm{R}_{1}$ and $\mathrm{R}_{2}$ be the dimensionless distance between the primaries. The equation of motion of the system with the minor modification (Khanna and Bhatnagar(1998)) and Usha et al.(2014)is given by:

$$
\begin{aligned}
\frac{\mathrm{d}^{2} \mathrm{x}}{\mathrm{dt}^{2}}=-\frac{1}{\mathrm{n}^{2}}\left[\frac{\mathrm{m}_{1} \mathrm{k}^{2}\left(\mathrm{x}-\mathrm{x}_{1}\right) \mathrm{q}_{1}}{\mathrm{R}_{1}{ }^{3}}+\frac{\mathrm{m}_{2} \mathrm{k}^{2}\left(\mathrm{x}-\mathrm{x}_{2}\right) \mathrm{q}_{2}}{\mathrm{R}_{2}{ }^{3}}\right. & \\
& +\frac{3 \mathrm{k}^{2} \mathrm{~m}_{1}\left(\mathrm{x}-\mathrm{x}_{1}\right)\left(2 \sigma_{1}-\sigma_{2}\right) \mathrm{q}_{1}}{2 \mathrm{R}_{1}{ }^{3} \mathrm{r}_{1}{ }^{2}} \\
& -\frac{15 \mathrm{k}^{2} \mathrm{~m}_{1}\left(\mathrm{x}-\mathrm{x}_{1}\right)\left(\sigma_{1}-\sigma_{2}\right) \mathrm{q}_{1} \mathrm{y}^{2}}{2 \mathrm{R}_{1}{ }^{3} \mathrm{r}_{1}{ }^{4}} \\
& +\frac{3 \mathrm{k}^{2} \mathrm{~m}_{2}\left(\mathrm{x}-\mathrm{x}_{2}\right)\left(2 \sigma_{1}{ }^{\prime}-\sigma_{2}{ }^{\prime}\right) \mathrm{q}_{2}}{2 \mathrm{R}_{2}{ }^{3} \mathrm{r}_{2}{ }^{2}} \\
& \left.-\frac{15 \mathrm{k}^{2} \mathrm{~m}_{2}\left(\mathrm{x}-\mathrm{x}_{2}\right)\left(\sigma_{1}{ }^{\prime}-\sigma_{2}{ }^{\prime}\right) \mathrm{q}_{2} \mathrm{y}^{2}}{2 \mathrm{R}_{2}{ }^{3} \mathrm{r}_{2}{ }^{4}}\right]
\end{aligned}
$$

$\frac{\mathrm{d}^{2} \mathrm{y}}{\mathrm{dt}^{2}}=$

$$
\begin{aligned}
& -\frac{1}{\mathrm{n}^{2}}\left[\frac{\mathrm{m}_{1} \mathrm{k}^{2}\left(\mathrm{y}-\mathrm{y}_{1}\right) \mathrm{q}_{1}}{\mathrm{R}_{1}{ }^{3}}+\frac{\mathrm{m}_{2} \mathrm{k}^{2}\left(\mathrm{y}-\mathrm{y}_{2}\right) \mathrm{q}_{2}}{\mathrm{R}_{2}{ }^{3}}+\frac{3 \mathrm{k}^{2} \mathrm{~m}_{1}\left(\mathrm{y}-\mathrm{y}_{1}\right)\left(2 \sigma_{1}-\sigma_{2}\right) \mathrm{q}_{1}}{2 \mathrm{R}_{1}{ }^{3} \mathrm{r}_{1}{ }^{2}}+\right. \\
& \frac{3 \mathrm{k}^{2} \mathrm{~m}_{1}\left(\mathrm{y}-\mathrm{y}_{1}\right)\left(\sigma_{1}-\sigma_{2}\right) \mathrm{q}_{1}}{2 \mathrm{R}_{1}{ }^{3} \mathrm{r}_{1}{ }^{2}}-\frac{15 \mathrm{k}^{2} \mathrm{~m}_{1}\left(\mathrm{y}-\mathrm{y}_{1}\right)\left(\sigma_{1}-\sigma_{2}\right) \mathrm{q}_{1} \mathrm{y}^{2}}{2 \mathrm{R}_{1}{ }^{3} \mathrm{r}_{1}{ }^{4}}- \\
& \frac{15 \mathrm{k}^{2} \mathrm{~m}_{2}\left(\mathrm{y}-\mathrm{y}_{2}\right)\left(\sigma_{1}{ }^{\prime}-\sigma_{2}{ }^{\prime}\right) \mathrm{q}_{2} \mathrm{y}^{2}}{2 \mathrm{R}_{2}{ }^{3} \mathrm{r}_{2}{ }^{4}}+\frac{3 \mathrm{k}^{2} \mathrm{~m}_{2}\left(\mathrm{y}-\mathrm{y}_{2}\right)\left(2 \sigma_{1}{ }^{\prime}-\sigma_{2}{ }^{\prime}\right)}{2 \mathrm{R}_{2}{ }^{3} \mathrm{r}_{2}{ }^{2}}+ \\
& \left.\frac{3 \mathrm{k}^{2} \mathrm{~m}_{2}\left(\mathrm{y}-\mathrm{y}_{2}\right)\left(\sigma_{1}{ }^{\prime}-\sigma_{2}{ }^{\prime}\right)}{\mathrm{R}_{2}{ }^{3} \mathrm{r}_{2}{ }^{2}}\right]
\end{aligned}
$$

Where

$$
\sigma_{1}=\left(A_{1}-A_{2}\right) ; \sigma_{2}=\left(A_{2}-A_{3}\right) ; A_{1}=\frac{a^{2}}{5 R^{2}} ; A_{2}=\frac{b^{2}}{5 R^{2}} ; A_{3}=\frac{c^{2}}{5 R^{2}}
$$

where $a, b, c$ are the axis of the triaxial rigid body of bigger primary of mass $m_{1}$. whereas $\sigma_{1}^{\prime}=\left(A_{1}^{\prime}-A_{2}^{\prime}\right) ; \sigma_{2}^{\prime}=\left(A_{2}^{\prime}-A_{3}^{\prime}\right)$; $\mathrm{A}_{1}^{\prime}=\frac{\mathrm{a}^{\prime 2}}{5 \mathrm{R}^{\prime 2}} ; \mathrm{A}_{2}^{\prime}=\frac{{\mathrm{b}^{\prime}}^{2}}{5 \mathrm{R}^{\prime 2}} ; \mathrm{A}_{3}^{\prime}=\frac{{\mathrm{c}^{\prime 2}}^{\prime^{\prime 2}}}{5 \mathrm{R}^{2}} \sigma_{2}$ where $\sigma_{1}, \sigma_{2}, \sigma_{1}^{\prime}$ and $\sigma_{2}^{\prime}$ are oblatenessparameter

wherea' $\mathrm{b}^{\prime}, \mathrm{c}^{\prime}$ are the axis of the triaxial rigid body of smaller primary of mass $m_{2}$. where $\mathrm{k}$ is the Gaussian constant, $\mathrm{t}^{*}$ is the dimensionless time, $\mathrm{q}_{1}$ and $\mathrm{q}_{2}$ are the parameters characterising the radiation pressure of primaries.

$$
\mathrm{n}^{2}=1+\frac{3}{2}\left(2 \mathrm{~A}_{1}-\mathrm{A}_{2}-\mathrm{A}_{3}\right)+\frac{3}{2}\left(2 \mathrm{~A}_{1}^{\prime}-\mathrm{A}_{2}^{\prime}-\mathrm{A}_{3}^{\prime}\right)
$$

where

$$
\mathrm{R}_{1}^{2}=\left(\mathrm{x}-\mathrm{x}_{1}\right)^{2}+\left(\mathrm{y}-\mathrm{y}_{1}\right)^{2}
$$

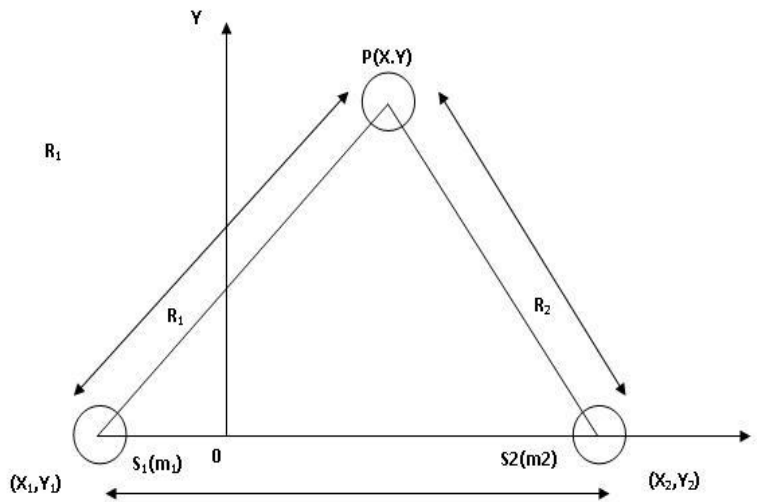

and

$\mathrm{R}_{2}^{2}=\left(\mathrm{x}-\mathrm{x}_{2}\right)^{2}+\left(\mathrm{y}-\mathrm{y}_{2}\right)^{2}$

We shall introduce a rotating co-ordinate system $(\bar{x}, \bar{y})$ by substituting

$$
\mathrm{Z}=\overline{\mathrm{z}} \mathrm{e}^{\mathrm{i} v}
$$

where $\mathrm{Z}=\mathrm{X}+\mathrm{iY}$

and $\bar{z}=\bar{x}+i \bar{y}$

After using the complex vector, the equation of motion (2.1) takes the form

$$
\begin{aligned}
& \frac{\mathrm{d}^{2} \mathrm{z}}{\mathrm{dt}^{*}{ }^{2}}= \\
& -\frac{1}{\mathrm{n}^{2}}\left[\frac{\mathrm{m}_{1} \mathrm{k}^{2}\left(\mathrm{z}-\mathrm{z}_{1}\right) \mathrm{q}_{1}}{\mathrm{R}_{1}{ }^{3}}+\frac{\mathrm{m}_{2} \mathrm{k}^{2}\left(\mathrm{z}-\mathrm{z}_{2}\right) \mathrm{q}_{2}}{\mathrm{R}_{2}{ }^{3}}+\frac{3 \mathrm{k}^{2} \mathrm{~m}_{1}\left(\mathrm{z}-\mathrm{z}_{1}\right)\left(2 \sigma_{1}-\sigma_{2}\right) \mathrm{q}_{1}}{2 \mathrm{R}_{1}{ }^{3} \mathrm{r}_{1}{ }^{2}}+\right. \\
& \frac{3 \mathrm{k}^{2} \mathrm{~m}_{2}\left(\mathrm{z}-\mathrm{z}_{2}\right)\left(2 \sigma_{1}{ }^{\prime}-\sigma_{2}{ }^{\prime}\right) \mathrm{q}_{2}}{2 \mathrm{R}_{2}{ }^{3} \mathrm{r}_{2}{ }^{2}}+\frac{3 \mathrm{k}^{2} \mathrm{~m}_{1}\left(\mathrm{z}^{*}-\mathrm{z}^{*}{ }^{*}\right)\left(\sigma_{1}-\sigma_{2}\right) \mathrm{q}_{1}}{\mathrm{R}_{1}{ }^{3} \mathrm{r}_{1}{ }^{2}}+ \\
& \frac{3 \mathrm{k}^{2} \mathrm{~m}_{2}\left(\mathrm{z}^{*}-\mathrm{z}^{*}\right)\left(\sigma_{1}{ }^{\prime}-\sigma_{2}{ }^{\prime}\right) \mathrm{q}_{2}}{\mathrm{R}_{2}{ }^{3} \mathrm{r}_{2}{ }^{2}{ }^{2}}-\frac{15 \mathrm{k}^{2} \mathrm{~m}_{1}\left(\mathrm{z}-\mathrm{z}_{1}\right)\left(\sigma_{1}-\sigma_{2}\right) \mathrm{q}_{1} \mathrm{y}^{2}}{2 \mathrm{R}_{1}{ }^{3} \mathrm{r}_{1}{ }^{4}}- \\
& \left.\frac{15 \mathrm{k}^{2} \mathrm{~m}_{2}\left(\mathrm{z}-\mathrm{z}_{2}\right)\left(\sigma_{1}{ }^{\prime}-\sigma_{2}{ }^{\prime}\right) \mathrm{q}_{2} \mathrm{y}^{2}}{2 \mathrm{R}_{2}{ }^{3} \mathrm{r}_{2}{ }^{4}}\right]
\end{aligned}
$$

where $\mathrm{z}^{*}=\mathrm{iy}, \mathrm{z}_{1}^{*}=\mathrm{iy}_{1}$ and $\mathrm{z}_{2}^{*}=\mathrm{iy}_{2}$. Now differentiating equation(2.3) twice with respect to $t^{*}$ and taking help of (2.4), we get:

$$
\begin{aligned}
& \frac{\mathrm{d}^{2} \mathrm{z}}{\mathrm{dt}^{* 2}}+2 \mathrm{i} \frac{\mathrm{d} v}{\mathrm{dt}^{*}} \cdot \frac{\mathrm{dz}}{\mathrm{dt}^{*}}=-\frac{1}{\mathrm{n}^{2}}\left[\frac{\mathrm{m}_{1} \mathrm{k}^{2}\left(\mathrm{z}-\mathrm{z}_{1}\right) \mathrm{q}_{1}}{\mathrm{R}_{1}{ }^{3}}+\frac{\mathrm{m}_{2} \mathrm{k}^{2}\left(\mathrm{z}-\mathrm{z}_{2}\right) \mathrm{q}_{2}}{\mathrm{R}_{2}{ }^{3}}+\right. \\
& \frac{3 \mathrm{k}^{2} \mathrm{~m}_{1}\left(\mathrm{z}-\mathrm{z}_{1}\right)\left(2 \sigma_{1}-\sigma_{2}\right) \mathrm{q}_{1}}{2 \mathrm{R}_{1}{ }^{3} \mathrm{r}_{1}{ }^{2}}+\frac{3 \mathrm{k}^{2} \mathrm{~m}_{2}\left(\mathrm{z}-\mathrm{z}_{2}\right)\left(2 \sigma_{1}{ }^{\prime}-\sigma_{2}{ }^{\prime}\right) \mathrm{q}_{2}}{2 \mathrm{R}_{2}{ }^{3} \mathrm{r}_{2}{ }^{2}}+ \\
& \frac{3 \mathrm{k}^{2} \mathrm{~m}_{1}\left(\mathrm{z}^{*}-\mathrm{z}^{*}\right)\left(\sigma_{1}-\sigma_{2}\right) \mathrm{q}_{1}}{\mathrm{R}_{1}{ }^{3} \mathrm{r}_{1}{ }^{2}}+\frac{3 \mathrm{k}^{2} \mathrm{~m}_{2}\left(\mathrm{z}^{*}-\mathrm{z}^{*}\right)\left(\sigma_{1}{ }^{\prime}-\sigma_{2}{ }^{\prime}\right) \mathrm{q}_{2}}{\mathrm{R}_{2}{ }^{3} \mathrm{r}_{2}{ }^{2}}- \\
& \left.\frac{15 \mathrm{k}^{2} \mathrm{~m}_{1}\left(\mathrm{z}-\mathrm{z}_{1}\right)\left(\sigma_{1}-\sigma_{2}\right) \mathrm{q}_{1} \mathrm{y}^{2}}{2 \mathrm{R}_{1}{ }^{3} \mathrm{r}_{1}{ }^{4}}-\frac{15 \mathrm{k}^{2} \mathrm{~m}_{2}\left(\mathrm{z}-\mathrm{z}_{2}\right)\left(\sigma_{1}{ }^{\prime}-\sigma_{2}\right) \mathrm{q}_{2} \mathrm{y}^{2}}{2 \mathrm{R}_{2}{ }^{3} \mathrm{r}_{2}{ }^{4}}\right]+\mathrm{z}\left(\frac{\mathrm{d} v}{\mathrm{dt}^{*}}\right)^{2}- \\
& \operatorname{iz} \frac{\mathrm{d}^{2} v}{\mathrm{dt}^{* 2}}
\end{aligned}
$$

In equation (2.5), the second term in the left hand side represents the Coriolis acceleration .The first term in the right hand side of the same equation is the gravitational effects due to radiation as well as triaxiality of the of the primaries .The second and third term represents the centrifugal effects and the acceleration normal to the radius vector due to non-uniform rotation of the system respectively.

The complex position vectors $\mathrm{z}_{1}$ and $\mathrm{z}_{2}$ are the location of the primaries, which are permanent on the real axis $(\bar{x}, \bar{y})$ of the system, we have:

$$
\mathrm{z}_{1}=\overline{\mathrm{x}_{1}}=\left(\frac{-\mathrm{p}_{1}}{1+\mathrm{e} \cos v}\right), \mathrm{z}_{2}=\overline{\mathrm{x}_{2}}=\left(\frac{\mathrm{p}_{2}}{1+\mathrm{e} \cos v}\right)
$$

where $\mathrm{p}_{1}$ and $\mathrm{p}_{2}$ are positive and

$\frac{\mathrm{p}_{1}}{\mathrm{p}_{2}}=\frac{\mathrm{a}_{1}}{\mathrm{a}_{2}}=\frac{\mathrm{m}_{2}}{\mathrm{~m}_{1}}$ 
where $a_{1}$ and $a_{2}$ are the semi major axis of the elliptical orbits of the massive primaries described about their centre of mass, and $\mathrm{m}_{1}, \mathrm{~m}_{2}$ are the masses of the respective bigger and smaller primaries. Further introducing a dimensionless pulsating coordinate system given by:

$\rho=\left(\frac{z}{r}\right)=x+i y$

where

$\mathrm{r}=\frac{\mathrm{a}\left(1-\mathrm{e}^{2}\right)}{1+\mathrm{ecos} v}$

Is the distance between the primaries in which a is a semi-major axis of the one primary around other. From equation (2.8) and (2.9), we get

$\mathrm{x}=\frac{\overline{\mathrm{x}}(1+\mathrm{ecos} v)}{\mathrm{a}\left(1-\mathrm{e}^{2}\right)}$

$y=\frac{\bar{y}(1+e \cos v)}{a\left(1-e^{2}\right)}$

Since these primaries are fixed in the coordinate system, we have form equation (2.6) and (2.10)

$\mathrm{x}_{1}=\frac{\overline{\mathrm{x}_{1}}(1+\mathrm{e} \cos v)}{\mathrm{a}\left(1-\mathrm{e}^{2}\right)}=\frac{-\mathrm{p}_{1}}{\mathrm{a}\left(1-\mathrm{e}^{2}\right)}=\frac{-\mathrm{a}_{1}}{\mathrm{a}}=-\mu$

$\mathrm{x}_{2}=\frac{\overline{\mathrm{x}_{2}}(1+\mathrm{e} \cos v)}{\mathrm{a}\left(1-\mathrm{e}^{2}\right)}=\frac{\mathrm{p}_{2}}{\mathrm{a}\left(1-\mathrm{e}^{2}\right)}=\frac{\mathrm{a}_{2}}{\mathrm{a}}=1-\mu$

Here $=\frac{m_{2}}{m_{1}+m_{2}}$. Therefore, the fixed location of these primaries in terms of coordinate $(x, y)$ system are represented as $(-\mu, 0)$ and $(1-\mu, 0)$.The coordinate taken into consideration regarding the problem of two bodies moving in the elliptical orbit are that ,the orbit of $m_{1}$ andm $m_{2}$ with respect to the centre of mass with semimajor axis is $\mathrm{a}_{1}=\mathrm{a} \mu$ and $\mathrm{a}_{2}=\mathrm{a}(1-\mu)$ respectively . Introducing true anomaly $v$ as an independent variable for solving the equation governing the motion of the system. From equation (2.8) we havez $=r \rho$, replacing

$\frac{m_{1} k^{2}\left(z-z_{1}\right) q_{1}}{R_{1}{ }^{3}}=\frac{m_{1} k^{2}\left(\rho-\rho_{1}\right) q_{1}}{r^{2} r_{1}{ }^{3}}$

where

$r_{1}^{2}=\left|\rho-\rho_{1}\right|^{2}=(x+\mu)^{2}+y^{2}$

Since

$$
\rho_{1}=x_{1}=-\mu
$$

$\frac{\mathrm{m}_{2} \mathrm{k}^{2}\left(\mathrm{z}-\mathrm{z}_{2}\right) \mathrm{q}_{2}}{\mathrm{R}_{2}{ }^{3}}=\frac{\mathrm{m}_{2} \mathrm{k}^{2}\left(\rho-\rho_{2}\right) \mathrm{q}_{2}}{\mathrm{r}^{2} \mathrm{r}_{2}{ }^{3}}$

where

$r_{2}^{2}=\left|\rho-\rho_{2}\right|^{2}=(x-1+\mu)^{2}+y^{2}$

Since

$$
\rho_{2}=x_{2}=1-\mu
$$

and

$\frac{3 k^{2} m_{1}\left(z-z_{1}\right)\left(2 \sigma_{1}-\sigma_{2}\right) q_{1}}{2 R_{1}^{3} r_{1}{ }^{2}}=\frac{3 k^{2} m_{1}\left(\rho-\rho_{1}\right)\left(2 \sigma_{1}-\sigma_{2}\right) q_{1}}{2 r^{2} r_{1}{ }^{5}}$

Similarly $\frac{3 k^{2} m_{2}\left(\mathrm{z}-\mathrm{z}_{2}\right)\left(2 \sigma_{1}{ }^{\prime}-\sigma_{2}{ }^{\prime}\right) q_{2}}{2 R_{2}{ }^{3} r_{2}{ }^{2}}=\frac{3 k^{2} m_{2}\left(\rho-\rho_{2}\right)\left(2 \sigma_{1}{ }^{\prime}-\sigma_{2}{ }^{\prime}\right) q_{2}}{2 r^{2} r_{2}{ }^{5}}$

Again

$\frac{3 k^{2} m_{1}\left(z^{*}-z^{*}\right)\left(\sigma_{1}-\sigma_{2}\right) q_{1}}{R_{1}{ }^{3} r_{1}{ }^{2}}=\frac{3 k^{2} m_{1}\left(\rho^{*}-\rho^{*}\right)\left(\sigma_{1}-\sigma_{2}\right) q_{1}}{r^{2} r_{1}{ }^{5}}$

Also

$\frac{3 k^{2} m_{2}\left(z^{*}-z^{*}\right)\left(\sigma_{1}{ }^{\prime}-\sigma_{2}{ }^{\prime}\right) q_{2}}{R_{2}{ }^{3} r_{2}{ }^{2}}=\frac{3 k^{2} m_{2}\left(\rho^{*}-\rho_{2}^{*}\right)\left(\sigma_{1}{ }^{\prime}-\sigma_{2}{ }^{\prime}\right) q_{2}}{r^{2} r_{2}{ }^{5}}$

Similarly

$\frac{15 k^{2} m_{1}\left(z-z_{1}\right)\left(\sigma_{1}-\sigma_{2}\right) q_{1} y^{2}}{2 R_{1}{ }^{3} r_{1}{ }^{4}}=\frac{15 k^{2} m_{1}\left(\rho-\rho_{1}\right)\left(\sigma_{1}-\sigma_{2}\right) q_{1} y^{2}}{2 r^{2} r_{1}{ }^{7}}$

And

$\frac{15 k^{2} m_{2}\left(z-z_{2}\right)\left(\sigma_{1}{ }^{\prime}-\sigma_{2}{ }^{\prime}\right) q_{2} y^{2}}{2 R_{2}{ }^{3} r_{2}{ }^{4}}$

Now taking $v$ as an independent variable, substituting all required above values in equation (2.5), we get:

$r\left(\frac{d v}{d t^{*}}\right)^{2}\left[\frac{d^{2} \rho}{d v^{2}}+2 i \frac{d \rho}{d v}\right]+\rho\left[\frac{d^{2} r}{d t^{* 2}}-r\left(\frac{d v}{d t^{*}}\right)^{2}\right]+\left(\frac{d \rho}{d v}+i \rho\right)$.

$\left[r \frac{d^{2} v}{d t^{* 2}}+2 \frac{d r}{d t^{*}} d v\right]=-\frac{1}{n^{2}}\left[\frac{m_{1} k^{2}\left(\rho-\rho_{1}\right) q_{1}}{r^{2} r_{1}{ }^{3}}+\frac{m_{2} k^{2}\left(\rho-\rho_{2}\right) q_{2}}{r^{2} r_{2}{ }^{3}}+\right.$

$\frac{3 k^{2} m_{1}\left(\rho-\rho_{1}\right)\left(2 \sigma_{1}-\sigma_{2}\right) q_{1}}{2 r^{2} r_{1}{ }^{5}}+\frac{3 k^{2} m_{2}\left(\rho-\rho_{2}\right)\left(2 \sigma_{1}{ }^{\prime}-\sigma_{2}{ }^{\prime}\right) q_{2}}{2 r^{2} r_{2}{ }^{5}}+$

$\frac{3 k^{2} m_{1}\left(\rho^{*}-\rho^{*}{ }_{1}\right)\left(\sigma_{1}-\sigma_{2}\right) q_{1}}{r^{2} r_{1}{ }^{5}}+\frac{3 k^{2} m_{2}\left(\rho^{*}-\rho^{*}{ }_{2}\right)\left(\sigma_{1}{ }^{\prime}-\sigma_{2}{ }^{\prime}\right) q_{2}}{r^{2} r_{2}{ }^{5}}-$

$\left.\frac{15 k^{2} m_{1}\left(\rho-\rho_{1}\right)\left(\sigma_{1}-\sigma_{2}\right) q_{1} y^{2}}{2 r^{2} r_{1}{ }^{7}}-\frac{15 k^{2} m_{2}\left(\rho-\rho_{2}\right)\left(\sigma_{1}{ }^{\prime}-\sigma_{2}{ }^{\prime}\right) q_{2} y^{2}}{2 r^{2} r_{2}{ }^{7}}\right]$

We have $=r(v)=\left[\frac{a\left(1-e^{2}\right)}{1+e \cos v}\right]$, which is the solution of two body problem involving the primaries $S_{1}$ and $S_{2}$. The integral of angular momentum of two body problem is given by:

$\left(r^{2} \frac{d v}{d t^{*}}\right)^{2}=a\left(1-e^{2}\right) k^{2}\left(m_{1}+m_{2}\right)$

Differentiating the above equation (2.22), with respect to $t^{*}$, we get:

$r \frac{d^{2} v}{d t^{* 2}}+2 \frac{d r}{d t^{*}} \frac{d v}{d t^{*}}=0$

The equation of motion of the two primaries is given by:

$\frac{d^{2} r}{d t^{* 2}}-r\left(\frac{d v}{d t^{*}}\right)^{2}=\frac{-k^{2}\left(m_{1}+m_{2}\right)}{r^{2}}$

Using equation (2.22), the equation (2.24) becomes:

$\frac{d^{2} r}{d t^{* 2}}-r\left(\frac{d v}{d t^{*}}\right)^{2}=\frac{-1}{a\left(1-e^{2}\right) r^{2}} \cdot\left(\frac{r^{2} d v}{d t^{*}}\right)^{2}$

Substituting the value of (2.23), the equation (2.25) becomes

$\frac{d^{2} r}{d t^{* 2}}-r\left(\frac{d v}{d t^{*}}\right)^{2}=\frac{-r^{2}}{a\left(1-e^{2}\right)} \cdot\left(\frac{d v}{d t^{*}}\right)^{2}$

Substituting the values of (2.23) and (2.26) in equation (2.21) we get

$\left(\frac{d^{2} \rho}{d v^{2}}\right)+2 i \frac{d \rho}{d v}=$

$\frac{r}{a\left(1-e^{2}\right)}[\rho-$

$\frac{1}{n^{2}}\left\{\frac{m_{1}}{m_{1}+m_{2}} \frac{\left(\rho-\rho_{1}\right) q_{1}}{r_{1}^{3}}+\frac{m_{2}}{m_{1}+m_{2}} \frac{\left(\rho-\rho_{2}\right) q_{2}}{r_{2}^{3}}+\frac{3 m_{1}}{m_{1}+m_{2}} \frac{\left(\rho-\rho_{1}\right)\left(2 \sigma_{1}-\sigma_{2}\right) q_{1}}{2 r_{1}^{5}}+\right.$ 


$$
\begin{aligned}
& \frac{3 m_{2}}{m_{1}+m_{2}} \frac{\left(\rho-\rho_{2}\right)\left(2 \sigma_{1}{ }^{\prime}-\sigma_{2}{ }^{\prime}\right) q_{2}}{2 r_{2}^{5}}+\frac{3 m_{1}}{m_{1}+m_{2}} \frac{\left(\rho^{*}-\rho^{*}{ }_{1}\right)\left(\sigma_{1}-\sigma_{2}\right) q_{1}}{r_{1}^{5}}+ \\
& \frac{3 m_{2}}{m_{1}+m_{2}} \frac{\left(\rho^{*}-\rho^{*}{ }_{2}\right)\left(\sigma_{1}{ }^{\prime}-\sigma_{2}{ }^{\prime}\right) q_{2}}{r_{2}^{5}}-\frac{15 m_{1}}{m_{1}+m_{2}} \frac{\left(\rho-\rho_{1}\right)\left(\sigma_{1}-\sigma_{2}\right) y^{2} q_{1}}{2 r_{1}^{7}}- \\
& \left.\left.\frac{15 m_{2}}{m_{1}+m_{2}} \frac{\left(\rho-\rho_{2}\right)\left(\sigma_{1}{ }^{\prime}-\sigma_{2}{ }^{\prime}\right) y^{2} q_{2}}{2 r_{2}^{7}}\right\}\right]
\end{aligned}
$$

\section{Using}

$r=\frac{a\left(1-e^{2}\right)}{1+e \cos v},=\frac{m_{2}}{m_{1}+m_{2}}, 1-\mu=\frac{m_{1}}{m_{1}+m_{2}}$,

The above expression will be reduced to the following form:

$\left(\frac{d^{2} \rho}{d v^{2}}\right)+2 i \frac{d \rho}{d v}=\frac{1}{1+e \cos v}\left[\rho-\frac{1}{n^{2}}\left\{\frac{(1-\mu)\left(\rho-\rho_{1}\right) q_{1}}{r_{1}^{3}}+\frac{\mu\left(\rho-\rho_{2}\right) q_{2}}{r_{2}^{3}}+\right.\right.$

$\frac{3(1-\mu)\left(\rho-\rho_{1}\right)\left(2 \sigma_{1}-\sigma_{2}\right) q_{1}}{2 r^{5}}+\frac{3 \mu\left(\rho-\rho_{2}\right)\left(2 \sigma_{1}{ }^{\prime}-\sigma_{2}{ }^{\prime}\right) q_{2}}{2 r^{5}}+$

$\frac{3(1-\mu)\left(\rho^{*}-\rho^{*}{ }_{1}\right)\left(\sigma_{1}-\sigma_{2}\right) q_{1}}{r_{1}^{5}}+\frac{3 \mu\left(\rho^{*}-\rho_{2}^{*}{ }_{2}\right)\left(\sigma_{1}{ }^{\prime}-\sigma_{2}{ }^{\prime}\right) q_{2}}{r_{2}^{5}}-$

$\left.\left.\frac{15(1-\mu)\left(\rho-\rho_{1}\right)\left(\sigma_{1}-\sigma_{2}\right) y^{2} q_{1}}{2 r_{1}^{7}}-\frac{15 \mu\left(\rho-\rho_{2}\right)\left(\sigma_{1}{ }^{\prime}-\sigma_{2}{ }^{\prime}\right) y^{2} q_{2}}{2 r_{2}^{7}}\right\}\right]$

Replacing

$\rho=x+i y, \rho_{1}=x_{1}+i y_{1}, \rho_{2}=x_{2}+i y_{2}, \rho^{*}=i y \quad, \rho_{1}^{*}=$ $i y_{1}, \rho_{2}^{*}=i y_{2}$

In the above Equation(2.28) and equating real and imaginary parts, we get:

$$
\begin{aligned}
\left(\frac{d^{2} x}{d v^{2}}\right)-2 \frac{d y}{d v}= & \frac{1}{1}+\operatorname{ecos} v[x \\
& -\frac{1}{n^{2}}\left\{\frac{(1-\mu)\left(x-x_{1}\right) q_{1}}{r_{1}^{3}}+\frac{\mu\left(x-x_{2}\right) q_{2}}{r_{2}^{3}}\right. \\
& +\frac{3(1-\mu)\left(x-x_{1}\right)\left(2 \sigma_{1}-\sigma_{2}\right) q_{1}}{2 r_{1}^{5}} \\
& +\frac{3 \mu\left(x-x_{2}\right)\left(2 \sigma_{1}{ }^{\prime}-\sigma_{2}{ }^{\prime}\right) q_{2}}{2 r_{2}^{5}} \\
& +-\frac{15(1-\mu)\left(x-x_{1}\right)\left(\sigma_{1}-\sigma_{2}\right) y^{2} q_{1}}{2 r_{1}^{7}} \\
& \left.\left.-\frac{15 \mu\left(x-x_{2}\right)\left(\sigma_{1}{ }^{\prime}-\sigma_{2}{ }^{\prime}\right) y^{2} q_{2}}{2 r_{2}^{7}}\right\}\right]
\end{aligned}
$$

And

$\left(\frac{d^{2} y}{d v^{2}}\right)+2 \frac{d x}{d v}=\frac{1}{1+e \cos v}\left[y-\frac{1}{n^{2}}\left\{\frac{(1-\mu)\left(y-y_{1}\right) q_{1}}{r_{1}^{3}}+\frac{\mu\left(y-y_{2}\right) q_{2}}{r_{2}^{3}}+\right.\right.$

$\frac{3(1-\mu)\left(y-y_{1}\right)\left(2 \sigma_{1}-\sigma_{2}\right) q_{1}}{2 r^{5}}+\frac{3 \mu\left(y-y_{2}\right)\left(2 \sigma_{1}{ }^{\prime}-\sigma_{2}{ }^{\prime}\right) q_{2}}{2 r^{5}}+$

$\frac{3(1-\mu)\left(y-y_{1}\right)\left(\sigma_{1}-\sigma_{2}\right) q_{1}}{r_{1}^{5}}+\frac{3 \mu\left(y-y_{2}\right)\left(\sigma_{1}{ }^{\prime}-\sigma_{2}{ }^{\prime}\right) q_{2}}{r_{2}^{5}}-$

$\left.\left.\frac{15(1-\mu)\left(y-y_{1}\right)\left(\sigma_{1}-\sigma_{2}\right) y^{2} q_{1}}{2 r_{1}^{7}}-\frac{15 \mu\left(y-y_{2}\right)\left(\sigma_{1}{ }^{\prime}-\sigma_{2}{ }^{\prime}\right) y^{2} q_{2}}{2 r_{2}^{7}}\right\}\right]$

Hence the equation of motion of infinitesimal shall be reduced to the following form by replacing

$x_{1}=-\mu, x_{2}=1-\mu, y_{1}=0, y_{2}=0$

$$
\begin{aligned}
\left(\frac{d^{2} x}{d v^{2}}\right)-2 \frac{d y}{d v}= & \frac{1}{1+e \operatorname{ecos} v}[x \\
& -\frac{1}{n^{2}}\left\{\frac{(1-\mu)(x+\mu) q_{1}}{r_{1}^{3}}+\frac{\mu(x-1+\mu) q_{2}}{r_{2}^{3}}\right. \\
& +\frac{3(1-\mu)(x+\mu)\left(2 \sigma_{1}-\sigma_{2}\right) q_{1}}{2 r_{1}^{5}} \\
& +\frac{3 \mu(x-1+\mu)\left(2 \sigma_{1}{ }^{\prime}-\sigma_{2}{ }^{\prime}\right) q_{2}}{2 r_{2}^{5}} \\
& -\frac{15(1-\mu)(x+\mu)\left(\sigma_{1}-\sigma_{2}\right) y^{2} q_{1}}{2 r_{1}^{7}} \\
& \left.\left.-\frac{15 \mu(x-1+\mu)\left(\sigma_{1}{ }^{\prime}-\sigma_{2}{ }^{\prime}\right) y^{2} q_{2}}{2 r_{2}^{7}}\right\}\right]
\end{aligned}
$$

and

$\left(\frac{d^{2} y}{d v^{2}}\right)+2 \frac{d x}{d v}=$

$\frac{1}{1+e \cos v}\left[y-\frac{1}{n^{2}}\left\{\frac{(1-\mu) y q_{1}}{r_{1}^{3}}+\frac{\mu y q_{2}}{r_{2}^{3}}+\frac{3(1-\mu) y\left(2 \sigma_{1}-\sigma_{2}\right) q_{1}}{2 r_{1}^{5}}+\right.\right.$

$\frac{3 \mu y\left(2 \sigma_{1}{ }^{\prime}-\sigma_{2}{ }^{\prime}\right) q_{2}}{2 r_{2}^{5}}+\frac{3(1-\mu) y\left(\sigma_{1}-\sigma_{2}\right) q_{1}}{r_{1}^{5}}+\frac{3 \mu y\left(\sigma_{1}{ }^{\prime}-\sigma_{2}{ }^{\prime}\right) q_{2}}{r_{2}^{5}}-$

$\left.\left.\frac{15(1-\mu)\left(\sigma_{1}-\sigma_{2}\right) y^{3} q_{1}}{2 r_{1}^{7}}-\frac{15 \mu\left(\sigma_{1}{ }^{\prime}-\sigma_{2}{ }^{\prime}\right) y^{3} q_{2}}{2 r_{2}^{7}}\right\}\right]$

The differential equation of motion of the third body $P$ in nondimensional barycentre, pulsating and non-uniformly rotating coordinate system $(x, y)$ is written in the form:

$x^{\prime \prime}-2 y^{\prime \prime}=\frac{1}{1+e \cos v}\left(\frac{\partial \Omega}{\partial x}\right)$

$y^{\prime \prime}+2 x^{\prime \prime}=\frac{1}{1+e \cos v}\left(\frac{\partial \Omega}{\partial y}\right)$

where ' denotes differentiation with respect to $v$, and

$\Omega=\frac{x^{2}+y^{2}}{2}+\frac{1}{n^{2}}\left[\frac{(1-\mu) q_{1}}{r_{1}}+\frac{\mu q_{2}}{r_{2}}+\frac{(1-\mu)\left(2 \sigma_{1}-\sigma_{2}\right) q_{1}}{2 r_{1}^{3}}+\frac{\mu\left(2 \sigma_{1}{ }^{\prime}-\sigma_{2}{ }^{\prime}\right) q_{2}}{2 r_{2}^{3}}-\right.$

$\frac{3(1-\mu)\left(\sigma_{1}-\sigma_{2}\right) y^{2} q_{1}}{2 r_{1}^{5}}-\frac{3 \mu\left(\sigma_{1}{ }^{\prime}-\sigma_{2}{ }^{\prime}\right) y^{2} q_{2}}{2 r_{2}^{5}}$

where

$n^{2}=1+\frac{3}{2}\left(2 \sigma_{1}-\sigma_{2}\right)+\frac{3}{2}\left(2 \sigma_{1}^{\prime}-\sigma_{2}^{\prime}\right)$

and

$$
r_{1}^{2}=(x+\mu)^{2}+y^{2}
$$

$$
r_{2}^{2}=(x-1+\mu)^{2}+y^{2}
$$

Thus we have the equation of motion of an elliptic restricted three body problem in which both the primaries are oblate, triaxial and radiating.

\section{Location of equilibrium points}

The equilibrium points of the system are the stationary points, so are given by the equation

$\frac{\partial \Omega}{\partial x}=\frac{\partial \Omega}{\partial y}=0$

Where $\Omega$ is given in equation (2.32), so we get:

$\frac{\partial \Omega}{\partial x}=x-\frac{1}{n^{2}}\left[\frac{(1-\mu)(x+\mu) q_{1}}{r_{1}^{3}}+\frac{\mu(x-1+\mu) q_{2}}{r_{2}^{3}}+\frac{3(1-\mu)(x+\mu)\left(2 \sigma_{1}-\sigma_{2}\right) q_{1}}{2 r_{1}^{5}}+\right.$ $\frac{3 \mu(x-1+\mu)\left(2 \sigma_{1}{ }^{\prime}-\sigma_{2}{ }^{\prime}\right) q_{2}}{2 r^{5}}-\frac{15(1-\mu)(x+\mu)\left(\sigma_{1}-\sigma_{2}\right) y^{2} q_{1}}{2 r^{7}}-$

$\left.\frac{15 \mu(x-1+\mu)\left(\sigma_{1}{ }^{\prime}-\sigma_{2}{ }^{\prime}\right) y^{2} q_{2}}{2 r_{2}^{7}}\right]=0$ 


\section{And}

$\frac{\partial \Omega}{\partial y}=y\left[1-\frac{1}{n^{2}}\left\{\frac{(1-\mu) q_{1}}{r_{1}^{3}}+\frac{\mu q_{2}}{r_{2}^{3}}+\frac{3(1-\mu)\left(4 \sigma_{1}-3 \sigma_{2}\right) q_{1}}{2 r_{1}^{5}}+\right.\right.$

$\left.\left.\frac{3 \mu\left(4 \sigma_{1}{ }^{\prime}-3 \sigma_{2}{ }^{\prime}\right) q_{2}}{2 r_{2}^{5}}-\frac{15(1-\mu)\left(\sigma_{1}-\sigma_{2}\right) y^{2} q_{1}}{2 r_{1}^{7}}-\frac{15 \mu\left(\sigma_{1}{ }^{\prime}-\sigma_{2}{ }^{\prime}\right) y^{2} q_{2}}{2 r_{2}^{7}}\right\}\right]$

Since $y \neq 0$,we have from equation(3.3):

$1-\frac{1}{n^{2}}\left[\frac{(1-\mu) q_{1}}{r_{1}^{3}}+\frac{\mu q_{2}}{r_{2}^{3}}+\frac{3(1-\mu)\left(4 \sigma_{1}-3 \sigma_{2}\right) q_{1}}{2 r_{1}^{5}}+\frac{3 \mu\left(4 \sigma_{1}{ }^{\prime}-3 \sigma_{2}{ }^{\prime}\right) q_{2}}{2 r_{2}^{5}}-\right.$

$\left.\frac{15(1-\mu)\left(\sigma_{1}-\sigma_{2}\right) y^{2} q_{1}}{2 r_{1}^{7}}-\frac{15 \mu\left(\sigma_{1}{ }^{\prime}-\sigma_{2}{ }^{\prime}\right) y^{2} q_{2}}{2 r_{2}^{7}}\right]=0$

Using the above equation (3.2) and (3.4), we get:

$\left[-n^{2}(1-\mu)+\frac{(1-\mu) q_{1}}{r_{1}^{3}}-\frac{15(1-\mu)\left(\sigma_{1}-\sigma_{2}\right) y^{2} q_{1}}{2 r_{1}^{7}}-\right.$

$\frac{15 \mu\left(\sigma_{1}{ }^{\prime}-\sigma_{2}{ }^{\prime}\right) y^{2} q_{2}}{2 r_{2}^{7}}+\frac{3(1-\mu)\left(2 \sigma_{1}-\sigma_{2}\right) q_{1}}{2 r_{1}^{5}}-\frac{3(1-\mu)(x-1+\mu)\left(\sigma_{1}-\sigma_{2}\right) q_{1}}{2 r_{1}^{5}}+$

$\left.\frac{3 \mu\left(2 \sigma_{1}{ }^{\prime}-\sigma_{2}{ }^{\prime}\right) q_{2}}{2 r_{2}^{5}}-\frac{3 \mu\left(\sigma_{1}{ }^{\prime}-\sigma_{2}{ }^{\prime}\right)(x-1+\mu) q_{2}}{r_{2}^{5}}\right]=0$

and

$-\mu+\frac{1}{n^{2}}\left[\frac{\mu q_{2}}{r_{2}^{3}}+\frac{3(1-\mu)(x+\mu)\left(\sigma_{1}-\sigma_{2}\right) q_{1}}{r_{1}^{5}}+\frac{3 \mu(x+\mu)\left(\sigma_{1}{ }^{\prime}-\sigma_{2}{ }^{\prime}\right) q_{2}}{r_{2}^{5}}+\right.$

$\left.\frac{3 \mu\left(2 \sigma_{1}{ }^{\prime}-\sigma_{2}{ }^{\prime}\right) q_{2}}{2 r_{2}^{5}}-\frac{15 \mu\left(\sigma_{1}{ }^{\prime}-\sigma_{2}{ }^{\prime}\right) y^{2} q_{2}}{2 r_{2}^{7}}\right]=0$

Since $\sigma_{1}, \sigma_{2}, \sigma_{1}{ }^{\prime}$ and $\sigma_{2}{ }^{\prime}$ are small quantities .If we take $\sigma_{1}=$ $\sigma_{2}=\sigma_{1}{ }^{\prime}=\sigma_{2}{ }^{\prime}=0$, the solution of the above equation is given by $r_{1}=r_{2}=1$ and $n=1$.Now we assume that the solution of the above equation, when $\sigma_{1} \neq 0, \sigma_{2} \neq 0, \sigma_{1}{ }^{\prime} \neq 0$ and $\sigma_{2}{ }^{\prime} \neq 0$ as

$r_{1}=1+\epsilon_{1}$

$r_{2}=1+\epsilon_{2}$

where $\epsilon_{1}$ and $\epsilon_{2}$ are less than 1 .

Substituting the values of $r_{1}$ and $r_{2}$ in equation (2.34) we get:

$x=\frac{1}{2}-\mu+\left(\epsilon_{1}+\epsilon_{2}\right)$

$y= \pm \frac{\sqrt{3}}{2}\left[1+\frac{2}{3}\left(\epsilon_{1}+\epsilon_{2}\right)\right]$

Substituting the values of $r_{1}$ and $r_{2}$ from equation (3.7) and $x$ and $y$ from (3.8) in equation (3.6), neglecting the higher order terms, we get:

$$
\begin{aligned}
& x=\frac{1}{2}-\mu+\frac{\left(\epsilon_{2}^{\prime}-\epsilon_{1}^{\prime}\right)}{3}+\left[\frac{-3}{8}-\epsilon_{1}^{\prime}-\frac{(1-\mu)}{2 \mu}+\frac{(1-\mu) \epsilon_{1}^{\prime}}{2 \mu}\right] \sigma_{1} \\
&+ {\left[\frac{7}{8}+\frac{\epsilon_{1}^{\prime}}{2}+\frac{(1-\mu)}{2 \mu}-\frac{\left.(1-\mu) \epsilon_{1}^{\prime}\right]}{2 \mu}\right] \sigma_{2} } \\
&+ {\left[\frac{3}{8}-\frac{3 \mu}{8(1-\mu)}+\frac{3 \mu \epsilon_{2}^{\prime}}{8(1-\mu)}+\epsilon_{2}^{\prime}\right] \sigma_{1}{ }^{\prime} } \\
&+ {\left[\frac{-7}{8}+\frac{7 \mu}{8(1-\mu)}-\frac{7 \mu \epsilon_{2}^{\prime}}{8(1-\mu)}-\frac{\epsilon_{2}^{\prime}}{2}\right] \sigma_{2}{ }^{\prime} }
\end{aligned}
$$

$y= \pm\left[1+\frac{2}{3}\left\{-\frac{\left(\epsilon_{1}^{\prime}+\epsilon_{2}^{\prime}\right)}{3}+\left[\frac{-19}{8}-\epsilon_{1}^{\prime}+\frac{(1-\mu)}{2 \mu}-\frac{(1-\mu) \epsilon_{1}^{\prime}}{2 \mu}\right] \sigma_{1}+\right.\right.$

$\left[\frac{15}{8}+\frac{\epsilon_{1}^{\prime}}{2}-\frac{(1-\mu)}{2 \mu}+\frac{(1-\mu) \epsilon_{1}^{\prime}}{2 \mu}\right] \sigma_{2}+\left[\frac{-19}{8}-\frac{3 \mu}{8(1-\mu)}+\frac{3 \mu \epsilon_{2}^{\prime}}{8(1-\mu)}-\right.$

$\left.\left.\left.\epsilon_{2}^{\prime}\right] \sigma_{1}{ }^{\prime}+\left[\frac{15}{8}+\frac{7 \mu}{8(1-\mu)}-\frac{7 \mu \epsilon_{2}^{\prime}}{8(1-\mu)}+\frac{\epsilon_{2}^{\prime}}{2}\right] \sigma_{2}{ }^{\prime}\right\}\right]$

\section{Stability of equilibrium points}

There are two triangular librations points of the problem of in the plane of finite bodies. Since the equilateral points are symmetrical to each other, the nature of motion near the two triangular libra- tions points will remain the same. Therefore, it is sufficient to analyze the motion of infinitesimal having the location $\left(x_{0}, y_{0}\right)$ given by the equation (3.9). In order to investigate the stability of the libration points (3.9) in the first approximation, deriving the variational equation of motion in the coordinates. Let, $\eta$ denote the small displacement in $\left(x_{0}, y_{0}\right)$. Then

$x=x_{0}+\xi$

$y=y_{0}+\eta$

Differentiating we get:

$x^{\prime}=\xi^{\prime}, y^{\prime}=\eta^{\prime}$

And

$x^{\prime \prime}=\xi^{\prime \prime}, y^{\prime \prime}=\eta^{\prime \prime}$

Now applying Taylor's theorem and retaining first order terms in the infinitesimal $\xi$ and, we get:

$\Omega_{x}=\Omega_{x}^{0}+\xi \Omega_{x x}^{0}+\eta \Omega_{x y}^{0}$

And

$\Omega_{y}=\Omega_{y}^{0}+\xi \Omega_{y x}^{0}+\eta \Omega_{y y}^{0}$

Here ,the subscript in $\Omega$ indicates the first and second partial derivatives as it appears once or twice, and the superscript 0 indicates that, the derivatives is to be evaluated around the equilibrium points $\left(x_{0}, y_{0}\right)$. Also, at the equilibrium points

$\Omega_{x}^{0}=\Omega_{y}^{0}=0$,

We have

$\Omega_{x}=\xi \Omega_{x x}^{0}+\eta \Omega_{x y}^{0}$

$\Omega_{y}=\xi \Omega_{y x}^{0}+\eta \Omega_{y y}^{0}$

Substituting the values from equation (4.2) and values of $x^{\prime}, y^{\prime}, x^{\prime \prime}, y^{\prime \prime}$ in equation (2.31), the equation of motion takes the form

$\xi^{\prime \prime}-2 \eta^{\prime}=\varnothing\left[\xi \Omega_{x x}^{0}+\eta \Omega_{x y}^{0}\right]$

$\eta^{\prime \prime}+2 \xi^{\prime}=\emptyset\left[\xi \Omega_{y x}^{0}+\eta \Omega_{y y}^{0}\right]$

Where $\emptyset=\left[\frac{1}{1+e \cos v}\right]$

Now, differentiating $\Omega$ partially with respect to $x, y$ respectively and evaluating $\Omega_{x x}, \Omega_{x y}$ and $\Omega_{y y}$ at the equilibrium points $\left(x_{0}, y_{0}\right)$ given by equation(3.9), we get:

$\Omega_{x x}^{0}=\frac{3}{4}+\frac{\epsilon_{2}^{\prime}}{2}-\frac{3 \mu \epsilon_{1}^{\prime}}{2}-\frac{3 \mu \epsilon_{2}^{\prime}}{2}+\sigma_{1}\left[\frac{-3}{8}+\frac{69 \mu}{16}+\frac{169 \epsilon_{1}^{\prime}}{32}-\frac{39 \mu \epsilon_{1}^{\prime}}{32}-\right.$

$\left.\frac{3}{4 \mu}+\frac{7 \epsilon_{1}^{\prime}}{4 \mu}\right]+\sigma_{2}\left[\frac{15}{8}-\frac{75 \mu}{16}-\frac{625 \epsilon_{1}^{\prime}}{32}+\frac{599 \mu \epsilon_{1}^{\prime}}{32}+\frac{3}{4 \mu}-\frac{7 \epsilon_{1}^{\prime}}{4 \mu}\right]+$

$\sigma_{1}^{\prime}\left[\frac{33}{16}-\frac{147 \mu}{32}+\frac{3 \epsilon_{2}^{\prime}}{16}+\frac{191 \mu \epsilon_{2}^{\prime}}{16}+\frac{9 \mu^{2}}{16(1-\mu)}-\frac{15 \mu^{2} \epsilon_{2}^{\prime}}{16(1-\mu)}\right]+$

$\sigma_{2}^{\prime}\left[\frac{-33}{16}+\frac{207 \mu}{32}-\frac{7 \epsilon_{2}^{\prime}}{16}-\frac{483 \mu \epsilon_{2}^{\prime}}{32}-\frac{21 \mu^{2}}{16(1-\mu)}+\frac{35 \mu^{2} \epsilon_{2}^{\prime}}{16(1-\mu)}\right]$

$\Omega_{x y}^{0}=\left\{\left(\frac{1}{2}-\mu-\frac{\epsilon_{1}^{\prime}}{9}-\frac{\mu \epsilon_{1}^{\prime}}{9}+\frac{2 \epsilon_{2}^{\prime}}{9}-\frac{14 \mu \epsilon_{2}^{\prime}}{9}\right)+\sigma_{1}\left[\frac{11}{24}-\frac{17 \mu}{24}+\right.\right.$ $\left.\frac{199 \epsilon_{1}^{\prime}}{48}-\frac{143 \mu \epsilon_{1}^{\prime}}{48}-\frac{1}{3 \mu}+\frac{17 \epsilon_{1}^{\prime}}{18 \mu}\right]+\sigma_{2}\left[\frac{3}{8}+\frac{13 \mu}{16}-\frac{557 \epsilon_{1}^{\prime}}{144}+\frac{445 \mu \epsilon_{1}^{\prime}}{144}+\right.$ $\left.\frac{1}{3 \mu}-\frac{17 \epsilon_{1}^{\prime}}{18 \mu}\right]+\sigma_{1}^{\prime}\left[\frac{7}{12}+\frac{31 \mu}{48}-\frac{161 \mu \epsilon_{2}^{\prime}}{36}-\frac{7 \epsilon_{2}^{\prime}}{6}-\frac{\mu^{2}}{4(1-\mu)}-\frac{\mu^{2} \epsilon_{2}^{\prime}}{12(1-\mu)}\right]+$ $\left.\sigma_{2}^{\prime}\left[\frac{-3}{4}-\frac{91 \mu}{48}+\frac{661 \mu \epsilon_{2}^{\prime}}{72}+\frac{47 \epsilon_{2}^{\prime}}{72}+\frac{7 \mu^{2}}{12(1-\mu)}+\frac{7 \mu^{2} \epsilon_{2}^{\prime}}{36(1-\mu)}\right]\right\}$ 
$\Omega_{y y}^{0}=\frac{9}{4}+\frac{\epsilon_{1}^{\prime}}{2}-\epsilon_{2}^{\prime}-\frac{3 \mu \epsilon_{1}^{\prime}}{2}+\frac{\mu \epsilon_{2}^{\prime}}{2}+\sigma_{1}\left[\frac{-21}{16}+\frac{849 \epsilon_{1}^{\prime}}{32}-\frac{25 \mu}{8}-\right.$

$\left.\frac{801 \mu \epsilon_{1}^{\prime}}{32}+\frac{3}{2 \mu}-\frac{3 \epsilon_{1}^{\prime}}{2 \mu}\right]+\sigma_{2}\left[\frac{33}{16}-\frac{837 \epsilon_{1}^{\prime}}{32}+\frac{21 \mu}{16}-+\frac{837 \mu \epsilon_{1}^{\prime}}{32}-\frac{3}{2 \mu}+\right.$

$\left.\frac{3 \epsilon_{1}^{\prime}}{2 \mu}\right]+\frac{\sigma_{1}^{\prime}}{(1-\mu)}\left[\frac{-21}{8}-\frac{183 \mu}{16}+\frac{8113 \mu \epsilon_{2}^{\prime}}{144}+\frac{603 \mu^{2}}{32}-\frac{7789 \mu^{2} \epsilon_{2}^{\prime}}{144}\right]+$

$\frac{\sigma_{2}^{\prime}}{(1-\mu)}\left[\frac{9}{4}-\frac{507 \mu}{16}-\frac{279 \mu \epsilon_{2}^{\prime}}{16}+\frac{549 \mu^{2}}{32}+\frac{195 \mu^{2} \epsilon_{2}^{\prime}}{16}\right]$

When eccentricity is neglected (i.e,$e=0$ ) the variational equation reduced directly to those for the circular restricted three body problem(CR3BP).

Now, in order to investigate the stability of the equilibrium points, new variables given by the equation below are introduced as:

$x_{1}=\xi, x_{2}=\eta, x_{3}=\frac{d \xi}{d v}, x_{4}=\frac{d \eta}{d v}$

Substituting these values in equation (4.3),the system of equations takes the form:

$\frac{d x_{i}}{d v}=P_{i 1} x_{1}+P_{i 2} x_{2}+P_{i 3} x_{3}+P_{i 4} x_{4} ; i=1,2,3,4$

Where

$$
\begin{gathered}
P_{11}=P_{12}=P_{13}=P_{14}=P_{22}=P_{23}=P_{33}=P_{44}=0 \\
P_{13}=1, P_{24}=1, P_{34}=2, P_{43}=-2
\end{gathered}
$$

We get:

$$
\begin{gathered}
P_{31}=\frac{1}{(1+e \cos v)} \Omega_{x x}^{0}=\phi \Omega_{x x}^{0} \\
P_{32}=P_{41}=\frac{1}{(1+e \cos v)} \Omega_{x y}^{0}=\phi \Omega_{x y}^{0}
\end{gathered}
$$

$P_{42}=\frac{1}{(1+e \cos v)} \Omega_{y y}^{0}=\phi \Omega_{y y}^{0}$

Where $\varnothing=\left[\frac{1}{1+e \cos v}\right]$. The coefficient in the system of equation (4.7) are the periodic functions of $v$ with period. Taking the average over the system, we get:

$\frac{d x_{i}^{(0)}}{d v}=P_{i 1}^{(0)} x_{1}^{(0)}+P_{i 2}^{(0)} x_{2}^{(0)}+=P_{i 3}^{(0)} x_{3}^{(0)}+=P_{i 4}^{(0)} x_{4}^{(0)}$

Where

$$
P_{i s}^{(0)}=\frac{1}{2 \pi} \int_{0}^{2 \pi} P_{i s}(v) d v, i, s=1,2,3,4
$$

From the formula (4.10), we obtain after evaluating the values:

$P_{11}^{(0)}=P_{12}^{(0)}=P_{14}^{(0)}=P_{21}^{(0)}=P_{22}^{(0)}=P_{23}^{(0)}=P_{33}^{(0)}=P_{44}^{(0)}=$

$0 ; P_{13}^{(0)}=1, P_{24}^{(0)}=1, P_{34}^{(0)}=2, P_{43}^{(0)}=-2$

$P_{31}^{(0)}=\frac{1}{\left(1-e^{2}\right)^{\frac{1}{2}}}\left[\frac{3}{4}+\frac{\epsilon_{2}^{\prime}}{2}-\frac{3 \mu \epsilon_{1}^{\prime}}{2}-\frac{3 \mu \epsilon_{2}^{\prime}}{2}+\sigma_{1}\left(\frac{-3}{8}+\frac{69 \mu}{16}+\frac{169 \epsilon_{1}^{\prime}}{32}-\right.\right.$

$\left.\frac{39 \mu \epsilon_{1}^{\prime}}{32}-\frac{3}{4 \mu}+\frac{7 \epsilon_{1}^{\prime}}{4 \mu}\right)+\sigma_{2}\left(\frac{15}{8}-\frac{75 \mu}{16}-\frac{625 \epsilon_{1}^{\prime}}{32}+\frac{599 \mu \epsilon_{1}^{\prime}}{32}+\frac{3}{4 \mu}-\frac{7 \epsilon_{1}^{\prime}}{4 \mu}\right)+$

$\sigma_{1}^{\prime}\left(\frac{33}{16}-\frac{147 \mu}{32}+\frac{3 \epsilon_{2}^{\prime}}{16}+\frac{191 \mu \epsilon_{2}^{\prime}}{16}+\frac{9 \mu^{2}}{16(1-\mu)}-\frac{15 \mu^{2} \epsilon_{2}^{\prime}}{16(1-\mu)}\right)+$

$\left.\sigma_{2}^{\prime}\left(\frac{-33}{16}+\frac{207 \mu}{32}-\frac{7 \epsilon_{2}^{\prime}}{16}-\frac{483 \mu \epsilon_{2}^{\prime}}{32}-\frac{21 \mu^{2}}{16(1-\mu)}+\frac{35 \mu^{2} \epsilon_{2}^{\prime}}{16(1-\mu)}\right)\right]$

Similarly

$$
\begin{aligned}
& P_{32}^{(0)}=P_{41}^{(0)}=\frac{1}{\left(1-e^{2}\right)^{\frac{1}{2}}} \frac{3 \sqrt{3}}{2}\left[\frac{1}{2}-\mu-\frac{\epsilon_{1}^{\prime}}{9}-\frac{\mu \epsilon_{1}^{\prime}}{9}+\frac{2 \epsilon_{2}^{\prime}}{9}-\frac{14 \mu \epsilon_{2}^{\prime}}{9}+\right. \\
& \sigma_{1}\left(\frac{11}{24}-\frac{17 \mu}{24}+\frac{199 \epsilon_{1}^{\prime}}{48}-\frac{143 \mu \epsilon_{1}^{\prime}}{48}-\frac{1}{3 \mu}+\frac{17 \epsilon_{1}^{\prime}}{18 \mu}\right)+\sigma_{2}\left(\frac{3}{8}+\frac{13 \mu}{16}-\right.
\end{aligned}
$$

$$
\begin{aligned}
& \left.\frac{557 \epsilon_{1}^{\prime}}{144}+\frac{445 \mu \epsilon_{1}^{\prime}}{144}+\frac{1}{3 \mu}-\frac{17 \epsilon_{1}^{\prime}}{18 \mu}\right)+\sigma_{1}^{\prime}\left(\frac{7}{12}+\frac{31 \mu}{48}-\frac{161 \mu \epsilon_{2}^{\prime}}{36}-\frac{7 \epsilon_{2}^{\prime}}{6}-\right. \\
& \left.\frac{\mu^{2}}{4(1-\mu)}-\frac{\mu^{2} \epsilon_{2}^{\prime}}{12(1-\mu)}\right)+\sigma_{2}^{\prime}\left(\frac{-3}{4}-\frac{91 \mu}{48}+\frac{661 \mu \epsilon_{2}^{\prime}}{72}+\frac{47 \epsilon_{2}^{\prime}}{72}+\frac{7 \mu^{2}}{12(1-\mu)}+\right. \\
& \left.\left.\frac{7 \mu^{2} \epsilon_{2}^{\prime}}{36(1-\mu)}\right)\right]
\end{aligned}
$$

And

$$
\begin{aligned}
& P_{42}^{(0)}=\frac{1}{\left(1-e^{2}\right)^{\frac{1}{2}}}\left[\frac{9}{4}+\frac{\epsilon_{1}^{\prime}}{2}-\epsilon_{2}^{\prime}-\frac{3 \mu \epsilon_{1}^{\prime}}{2}+\frac{\mu \epsilon_{2}^{\prime}}{2}+\sigma_{1}\left(\frac{-21}{16}+\frac{849 \epsilon_{1}^{\prime}}{32}-\right.\right. \\
& \left.\frac{25 \mu}{8}-\frac{801 \mu \epsilon_{1}^{\prime}}{32}+\frac{3}{2 \mu}-\frac{3 \epsilon_{1}^{\prime}}{2 \mu}\right)+\sigma_{2}\left(\frac{33}{16}-\frac{837 \epsilon_{1}^{\prime}}{32}+\frac{21 \mu}{16}-+\frac{837 \mu \epsilon_{1}^{\prime}}{32}-\right. \\
& \left.\frac{3}{2 \mu}+\frac{3 \epsilon_{1}^{\prime}}{2 \mu}\right)+\sigma_{1}^{\prime}\left(\frac{-21}{8(1-\mu)}-\frac{183 \mu}{16(1-\mu)}+\frac{8113 \mu \epsilon_{2}^{\prime}}{144(1-\mu)}+\frac{603 \mu^{2}}{32(1-\mu)}-\right. \\
& \left.\frac{7789 \mu^{2} \epsilon_{2}^{\prime}}{144(1-\mu)}+\sigma_{2}^{\prime}\left(\begin{array}{c}
\frac{9}{4(1-\mu)}-\frac{507 \mu}{16(1-\mu)}-\frac{279 \mu \epsilon_{2}^{\prime}}{16(1-\mu)} \\
+\frac{549 \mu^{2}}{32(1-\mu)}+\frac{195 \mu^{2} \epsilon_{2}^{\prime}}{16(1-\mu)}
\end{array}\right)\right]
\end{aligned}
$$

The system of equation in the first approximation is represented as follows:

The characteristic equation for the system is:

$\lambda^{4}-Q \lambda^{2}+R=0$

Where,

$Q=P_{31}^{(0)}+P_{42}^{(0)}-4$

And $R=P_{31}^{(0)} P_{42}^{(0)}-P_{32}^{(0)} P_{41}^{(0)}$

The roots of characteristic equation(4.15) is given by

$\lambda=\left[\frac{Q}{2}+\frac{\left(Q^{2}-4 R\right)^{\frac{1}{2}}}{2}\right]^{\frac{1}{2}}$

The characteristic roots will be purely imaginary if

$Q<0$

And

$Q^{2}-4 R \geq 0$

From Equation (4.18) it follows that: 


$$
\begin{aligned}
\frac{1}{\left(1-e^{2}\right)^{\frac{1}{2}}\left[3+\frac{\epsilon_{1}^{\prime}}{2}-\right.} & \frac{\epsilon_{2}^{\prime}}{2}-3 \mu \epsilon_{1}^{\prime}-\mu \epsilon_{2}^{\prime} \\
& +\sigma_{1}\left(\frac{-27}{16}+\frac{19 \mu}{16}+\frac{509 \epsilon_{1}^{\prime}}{16}-\frac{105 \mu \epsilon_{1}^{\prime}}{4}+\frac{3}{4 \mu}\right. \\
& \left.+\frac{3 \epsilon_{1}^{\prime}}{4 \mu}\right) \\
& +\sigma_{2}\left(\frac{63}{16}-\frac{27 \mu}{8}-\frac{731 \epsilon_{1}^{\prime}}{16}+\frac{359 \mu \epsilon_{1}^{\prime}}{8}-\frac{3}{4 \mu}\right. \\
& \left.-\frac{\epsilon_{1}^{\prime}}{4 \mu}\right) \\
& +\sigma_{1}^{\prime}\left(\frac{33}{16}-\frac{147 \mu}{32}+\frac{3 \epsilon_{2}^{\prime}}{16}+\frac{191 \mu \epsilon_{2}^{\prime}}{16}\right. \\
& +\frac{621 \mu^{2}}{32(1-\mu)}-\frac{1981 \mu^{2} \epsilon_{2}^{\prime}}{36(1-\mu)}-\frac{21}{8(1-\mu)} \\
& \left.-\frac{183 \mu}{16(1-\mu)}+\frac{8113 \mu \epsilon_{2}^{\prime}}{144(1-\mu)}\right) \\
& +\sigma_{2}^{\prime}\left(\frac{-33}{16}+\frac{207 \mu}{32}-\frac{7 \epsilon_{2}^{\prime}}{16}-\frac{483 \mu \epsilon_{2}^{\prime}}{32}\right. \\
& +\frac{507 \mu^{2}}{32(1-\mu)}+\frac{115 \mu^{2} \epsilon_{2}^{\prime}}{8(1-\mu)}+\frac{9}{4(1-\mu)} \\
& \left.\left.-\frac{507 \mu}{16(1-\mu)}-\frac{279 \mu \epsilon_{2}^{\prime}}{16(1-\mu)}\right)-4\left(1-e^{2}\right)^{\frac{1}{2}}\right] \\
& <0
\end{aligned}
$$

It is clear when $\sigma_{1}, \sigma_{2}, \sigma_{1}^{\prime}, \sigma_{2}^{\prime}, \epsilon_{1}^{\prime}, \epsilon_{2}^{\prime}$ are zero, we get

$$
0<e<0.661437827
$$

The behaviour of the system can be investigated when the infinitesimal mass moves around the triangular equilibrium points under the radiating oblate triaxial primaries by plotting the transition curves for the different values of $e$ and the parameters $\sigma_{1}, \sigma_{2}, \sigma_{1}^{\prime}, \sigma_{2}^{\prime}$ and the radiation parameter $\epsilon_{1}^{\prime}$ and $\epsilon_{2}^{\prime}$.Using MATLAB 7.11.0 version, we have plotted curves between different values of eccentricity of the orbit of infinitesimal and $\mu^{*}$ (critical mass ratio) by varying radiation parameters and $\sigma_{1}, \sigma_{2}$ and $\sigma_{1}^{\prime}, \sigma_{2}^{\prime}$ shown by the figure(1-10),maintaining the condition $\left|\sigma_{1}-\sigma_{2}\right|>0$ and $\left|\sigma_{1}^{\prime}-\sigma_{2}^{\prime}\right|>0$.

We observe that the increment in the radiation parameter attributes to the decrease in the region of stability of the infinitesimal around the triangular equilibrium points which is obvious from the figures. Also,the increase in the difference $\left|\sigma_{1}-\sigma_{2}\right|>0$ and increase in the $\left|\sigma_{1}^{\prime}-\sigma_{2}^{\prime}\right|>0$ has also a destabilizing effect which is also obvious from the figures.

In case the eccentricity $e$ does not satisfy inequality (4.20), the characteristic roots will either real or complex conjugate. In the case with complex roots, there are roots with positive real parts, causing equilibrium points to be unstable in the first approximation.

From the inequality (4.19), we obtain:

$\frac{1}{\left(1-e^{2}\right)}\left[A \mu^{2}+B \mu+C\right] \geq 0$

Where

$$
\begin{aligned}
A=\left\{27+\frac{153 \sigma_{1}}{8}\right. & -\frac{351 \sigma_{2}}{8}-\frac{495 \sigma_{1}^{\prime}}{32}+\frac{6669 \sigma_{2}^{\prime}}{4}+3 \epsilon_{1}^{\prime}+84 \epsilon_{2}^{\prime} \\
& +\frac{1321 \sigma_{1} \epsilon_{1}^{\prime}}{8}-\frac{687 \sigma_{2} \epsilon_{1}^{\prime}}{4}+\frac{12835 \sigma_{1}^{\prime} \epsilon_{2}^{\prime}}{96} \\
& -487 \sigma_{2}^{\prime} \epsilon_{2}^{\prime} \\
& +\left(1-e^{2}\right)^{1 / 2}\left[\frac{111 \sigma_{1}^{\prime}}{4}-\frac{507 \sigma_{2}^{\prime}}{4}-\frac{21 \sigma_{1}^{\prime} \epsilon_{2}^{\prime}}{2}\right. \\
& \left.\left.+\frac{49 \sigma_{2}^{\prime} \epsilon_{2}^{\prime}}{2}\right]\right\}
\end{aligned}
$$

$$
\begin{aligned}
& B=\left\{-27-\frac{1059 \sigma_{1}}{16}+\frac{477 \sigma_{2}}{16}-\frac{1359 \sigma_{1}^{\prime}}{32}-\frac{3789 \sigma_{2}^{\prime}}{32}+9 \epsilon_{1}^{\prime}\right. \\
& -48 \epsilon_{2}^{\prime}-\frac{693 \sigma_{1} \epsilon_{1}^{\prime}}{4}+\frac{5001 \sigma_{2} \epsilon_{1}^{\prime}}{16}-\frac{5425 \sigma_{1}^{\prime} \epsilon_{2}^{\prime}}{48} \\
& +\frac{9793 \sigma_{2}^{\prime} \epsilon_{2}^{\prime}}{32} \\
& +\left(1-e^{2}\right)^{1 / 2}\left[-\frac{19 \sigma_{1}}{2}+\frac{27 \sigma_{2}}{2}+\frac{963 \sigma_{1}^{\prime}}{4}\right. \\
& +\frac{735 \sigma_{2}^{\prime}}{4}+24 \epsilon_{1}^{\prime}+8 \epsilon_{2}^{\prime}+210 \sigma_{1} \epsilon_{1}^{\prime} \\
& \left.\left.-359 \sigma_{2} \epsilon_{1}^{\prime}-\frac{4916 \sigma_{1}^{\prime} \epsilon_{2}^{\prime}}{9}+\frac{1041 \sigma_{2}^{\prime} \epsilon_{2}^{\prime}}{4}\right]\right\} \\
& C=\left\{25-16 e^{2}-24\left(1-e^{2}\right)^{1 / 2}+\frac{441 \sigma_{1}}{16}-\frac{1177 \sigma_{2}}{4}+\frac{27 \sigma_{1}^{\prime}}{16}\right. \\
& +\frac{2169 \sigma_{2}^{\prime}}{8}+\frac{9 \epsilon_{1}^{\prime}}{2}+\frac{3 \epsilon_{2}^{\prime}}{2}+\frac{327 \sigma_{1} \epsilon_{1}^{\prime}}{4} \\
& -\frac{253 \sigma_{2} \epsilon_{1}^{\prime}}{8}-11 \sigma_{1}^{\prime} \epsilon_{2}^{\prime}+\frac{3 \sigma_{2}^{\prime} \epsilon_{2}^{\prime}}{8} \\
& +\left(1-e^{2}\right)^{1 / 2}\left[\frac{27 \sigma_{1}}{2}-\frac{63 \sigma_{2}}{2}+\frac{9 \sigma_{1}^{\prime}}{2}-\frac{3 \sigma_{2}^{\prime}}{2}\right. \\
& -12 \epsilon_{1}^{\prime}+4 \epsilon_{2}^{\prime}-\frac{509 \sigma_{1} \epsilon_{1}^{\prime}}{2}+\frac{731 \sigma_{2} \epsilon_{2}^{\prime}}{2} \\
& \left.\left.-\frac{3 \sigma_{1}^{\prime} \epsilon_{2}^{\prime}}{2}+\frac{7 \sigma_{2}^{\prime} \epsilon_{2}^{\prime}}{2}\right]\right\}
\end{aligned}
$$

Since $\leq \frac{1}{2}$, the inequality is satisfied for $0 \leq \mu<\mu^{*}$, where $\mu^{*}$ is given by

$$
\mu^{*}=\frac{-N_{1} \pm N_{2}^{1 / 2}}{2 A}
$$

And the value of $N_{1}$ and $N_{2}=B^{2}-4 A C$, are given by the following equation:

$$
\begin{aligned}
N_{1}=\left\{-27-\frac{1059 \sigma_{1}}{16}+\frac{477 \sigma_{2}}{16}-\frac{1359 \sigma_{1}^{\prime}}{32}-\frac{3789 \sigma_{2}^{\prime}}{32}+9 \epsilon_{1}^{\prime}\right. \\
-48 \epsilon_{2}^{\prime}-\frac{693 \sigma_{1} \epsilon_{1}^{\prime}}{4}+\frac{5001 \sigma_{2} \epsilon_{1}^{\prime}}{16}-\frac{5425 \sigma_{1}^{\prime} \epsilon_{2}^{\prime}}{48} \\
+\frac{9793 \sigma_{2}^{\prime} \epsilon_{2}^{\prime}}{32} \\
+\left(1-e^{2}\right)^{1 / 2}\left[-\frac{19 \sigma_{1}}{2}+\frac{27 \sigma_{2}}{2}+\frac{963 \sigma_{1}^{\prime}}{4}\right. \\
+\frac{735 \sigma_{2}^{\prime}}{4}+24 \epsilon_{1}^{\prime}+8 \epsilon_{2}^{\prime}+210 \sigma_{1} \epsilon_{1}^{\prime} \\
\left.\left.-359 \sigma_{2} \epsilon_{1}^{\prime}-\frac{4916 \sigma_{1}^{\prime} \epsilon_{2}^{\prime}}{9}+\frac{1041 \sigma_{2}^{\prime} \epsilon_{2}^{\prime}}{4}\right]\right\}
\end{aligned}
$$

$N_{2}=\left[\left\{-27-\frac{1059 \sigma_{1}}{16}+\frac{477 \sigma_{2}}{16}-\frac{1359 \sigma_{1}^{\prime}}{32}-\frac{3789 \sigma_{2}^{\prime}}{32}+9 \epsilon_{1}^{\prime}-\right.\right.$ $48 \epsilon_{2}^{\prime}-\frac{693 \sigma_{1} \epsilon_{1}^{\prime}}{4}+\frac{5001 \sigma_{2} \epsilon_{1}^{\prime}}{16}-\frac{5425 \sigma_{1}^{\prime} \epsilon_{2}^{\prime}}{48}+\frac{9793 \sigma_{2}^{\prime} \epsilon_{2}^{\prime}}{32}+(1-$ $\left.e^{2}\right)^{1 / 2}\left[-\frac{19 \sigma_{1}}{2}+\frac{27 \sigma_{2}}{2}+\frac{963 \sigma_{1}^{\prime}}{4}+\frac{735 \sigma_{2}^{\prime}}{4}+24 \epsilon_{1}^{\prime}+8 \epsilon_{2}^{\prime}+\right.$ $\left.\left.210 \sigma_{1} \epsilon_{1}^{\prime}-359 \sigma_{2} \epsilon_{1}^{\prime}-\frac{4916 \sigma_{1}^{\prime} \epsilon_{2}^{\prime}}{9}+\frac{1041 \sigma_{2}^{\prime} \epsilon_{2}^{\prime}}{4}\right]\right\}^{2}-4\{27+$ $\frac{153 \sigma_{1}}{8}-\frac{351 \sigma_{2}}{8}-\frac{495 \sigma_{1}^{\prime}}{32}+\frac{6669 \sigma_{2}^{\prime}}{4}+3 \epsilon_{1}^{\prime}+84 \epsilon_{2}^{\prime}+\frac{1321 \sigma_{1} \epsilon_{1}^{\prime}}{8}-$ $\frac{687 \sigma_{2} \epsilon_{1}^{\prime}}{4}+\frac{12835 \sigma_{1}^{\prime} \epsilon_{2}^{\prime}}{96}-487 \sigma_{2}^{\prime} \epsilon_{2}^{\prime}+\left(1-e^{2}\right)^{1 / 2}\left[\frac{111 \sigma_{1}^{\prime}}{4}-\frac{507 \sigma_{2}^{\prime}}{4}-\right.$ $\left.\left.\frac{21 \sigma_{1}^{\prime} \epsilon_{2}^{\prime}}{2}+\frac{49 \sigma_{2}^{\prime} \epsilon_{2}^{\prime}}{2}\right]\right\} \cdot\left\{25-16 e^{2}-24\left(1-e^{2}\right)^{1 / 2}+\frac{441 \sigma_{1}}{16}-\right.$ $\frac{1177 \sigma_{2}}{4}+\frac{27 \sigma_{1}^{\prime}}{16}+\frac{2169 \sigma_{2}^{\prime}}{8}+\frac{9 \epsilon_{1}^{\prime}}{2}+\frac{3 \epsilon_{2}^{\prime}}{2}+\frac{327 \sigma_{1} \epsilon_{1}^{\prime}}{4}-\frac{253 \sigma_{2} \epsilon_{1}^{\prime}}{8}-$ $11 \sigma_{1}^{\prime} \epsilon_{2}^{\prime}+\frac{3 \sigma_{2}^{\prime} \epsilon_{2}^{\prime}}{8}+\left(1-e^{2}\right)^{1 / 2}\left[\frac{27 \sigma_{1}}{2}-\frac{63 \sigma_{2}}{2}+\frac{9 \sigma_{1}^{\prime}}{2}-\frac{3 \sigma_{2}^{\prime}}{2}-12 \epsilon_{1}^{\prime}+\right.$ $\left.\left.\left.4 \epsilon_{2}^{\prime}-\frac{509 \sigma_{1} \epsilon_{1}^{\prime}}{2}+\frac{731 \sigma_{2} \epsilon_{2}^{\prime}}{2}-\frac{3 \sigma_{1}^{\prime} \epsilon_{2}^{\prime}}{2}+\frac{7 \sigma_{2}^{\prime} \epsilon_{2}^{\prime}}{2}\right]\right\}\right](4.22)$ 
Thus the triangular equilibrium points are stable if the eccentricity ' $e$ ' satisfies the condition (4.20) and the mass ratio $\mu^{*}$ obeys the (4.22). The dependence of $\mu^{*}$ on the eccentricity $e$ are plotted in the graphs for different values of oblate triaxial parameters $\sigma_{1}, \sigma_{2}$, $\sigma_{1}^{\prime}$ and $\sigma_{2}^{\prime}$ and $\epsilon_{1}^{\prime}, \epsilon_{2}^{\prime}$,where $\epsilon_{1}^{\prime}, \epsilon_{2}^{\prime}$ are radiation pressures. It is observed that with the increase in the radiation pressure the range of stability decreases and also, the increase in the difference $\mid \sigma_{1}-$ $\sigma_{2} \mid$ and $\left|\sigma_{1}^{\prime}-\sigma_{2}^{\prime}\right|$ have a destabilizing effect.

\section{Discussion and conclusion}

The equation of motion of an infinitesimal particle has been derived under the assumption that the primaries are oblate triaxial and the source of radiations moving around each other about their centre of mass in their elliptical orbits. These equations are effected by radiation pressure, oblate triaxiality, semi major axis and eccentricity of orbits of the primaries. The triangular equilibrium points shifted away from the axis with increase in radiation factors.also the stability has been investigated and is found that triangular points are stable if eccentricity satisfies the defined condition represented by (4.20) and the mass ratio obeys (4.22) where $\mu^{*}$ depends on effects of the parameters involved. It is further observed that all the parameters affect the stability as follows:

1) The region of stability decreases with the increase in the radiation pressure, (Figs 1-5)when $\left|\sigma_{1}-\sigma_{2}\right|$ and $\left|\sigma_{1}^{\prime}-\sigma_{2}^{\prime}\right|$ are grater than Zero, it is desterilizing effects (Figs 6-10).

2) When $\left|\sigma_{1}-\sigma_{2}\right|=0$ and $\left|\sigma_{1}^{\prime}-\sigma_{2}^{\prime}\right|=0$ that is when the primaries are perfectly spherical then radiation predominates.

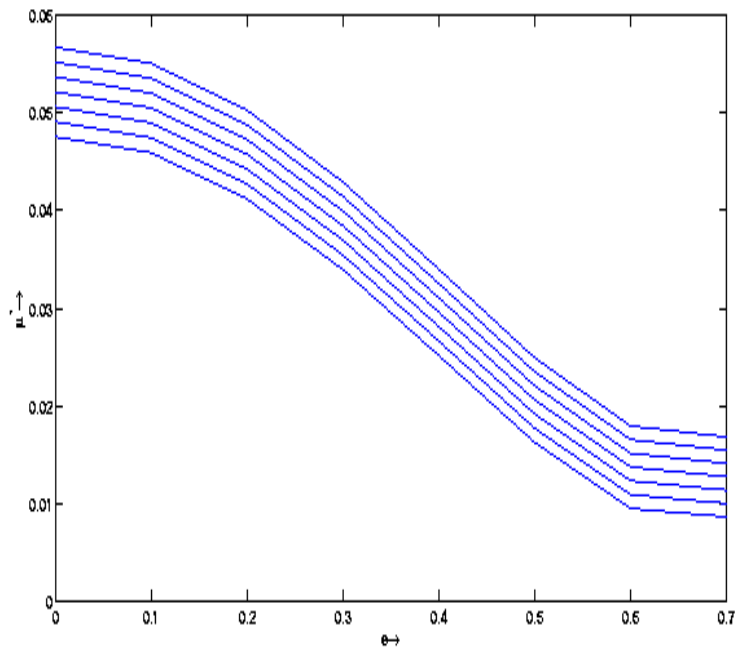

Fig.1:Transition Curves in $\mu^{*}$ and $e$ for $\sigma_{1}=0.003$ to $0.009, \sigma_{2}=0.0002$, $\sigma_{1}^{\prime}=0.0007, \sigma_{2}^{\prime}=0.0004, \epsilon_{1}^{\prime}=0.0001, \epsilon_{2}^{\prime}=0.0002$.

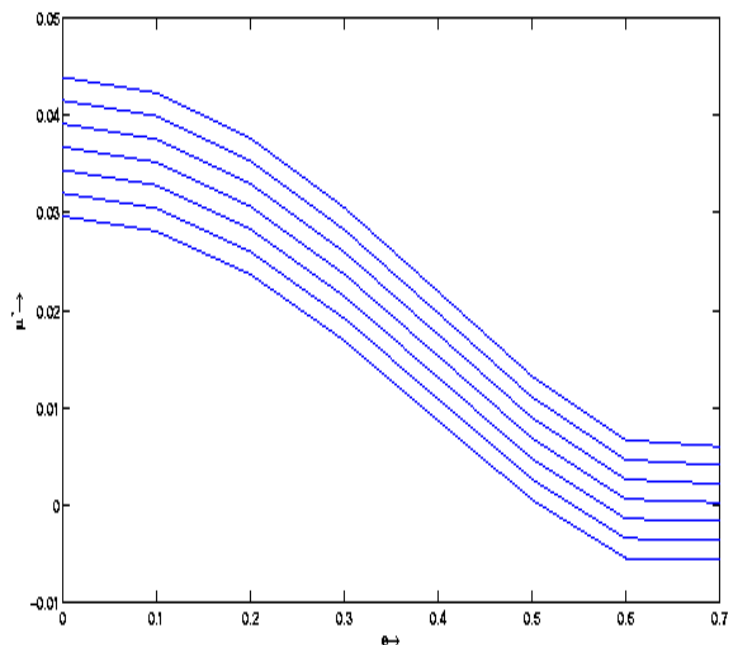

Fig.2: Transition Curves in $\mu^{*}$ and $e$ for $\sigma_{1}=0.005, \sigma_{2}=0.003$ to 0.009 $\sigma_{1}^{\prime}=0.0007, \sigma_{2}^{\prime}=0.0004, \epsilon_{1}^{\prime}=0.0001, \epsilon_{2}^{\prime}=0.0002$.

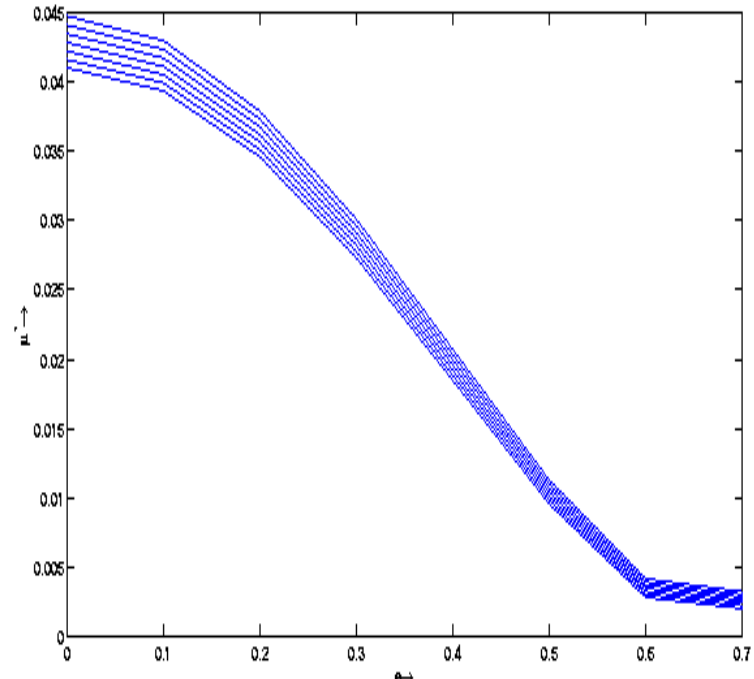

Fig.3: Transition Curves in $\mu^{*}$ and $e$ for $\sigma_{1}=0.0005, \sigma_{2}=0.0002, \sigma_{1}^{\prime}=$ 0.003 to $0.009, \sigma_{2}^{\prime}=0.0004, \epsilon_{1}^{\prime}=0.0001, \epsilon_{2}^{\prime}=0.0002$.

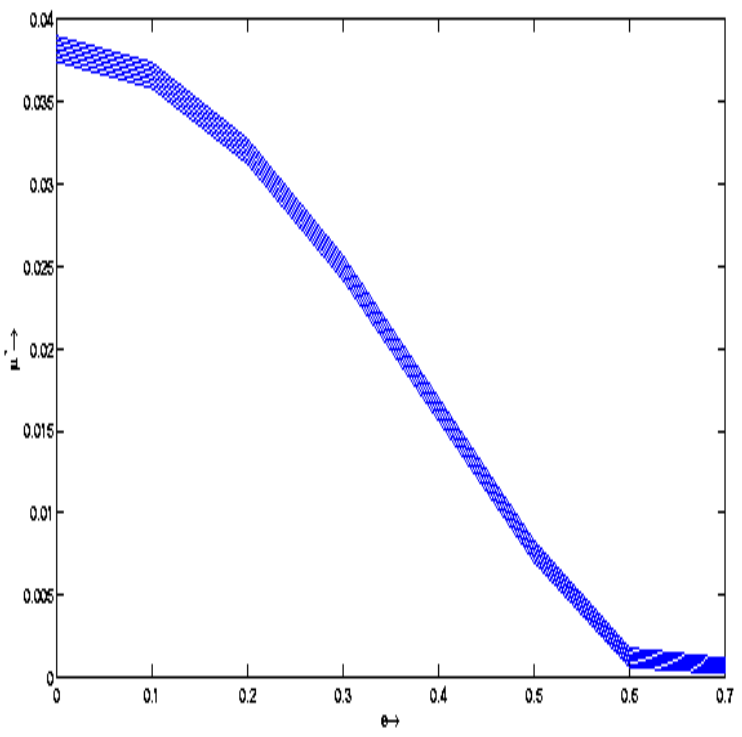

Fig.4:Transition Curves in $\mu^{*}$ and $e$ for $\sigma_{1}=0.0005, \sigma_{2}=0.0002$, $\sigma_{1}^{\prime}=0.0007, \sigma_{2}^{\prime}=0.0004, \epsilon_{1}^{\prime}=0.003$ to $0.009, \epsilon_{2}^{\prime}=0.0002$.

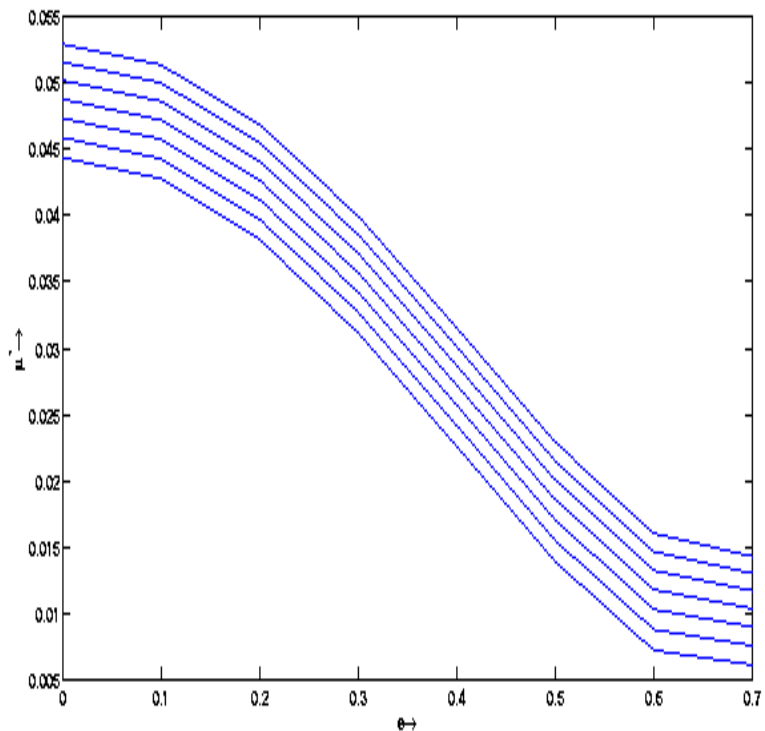

Fig.5:Transition Curves in $\mu^{*}$ and $e$ for $\sigma_{1}=0.0005, \sigma_{2}=0.0002, \sigma_{1}^{\prime}=$ $0.0007, \sigma_{2}^{\prime}=0.0004, \epsilon_{1}^{\prime}=0.00001, \epsilon_{2}^{\prime}=0.003$ to 0.009 . 


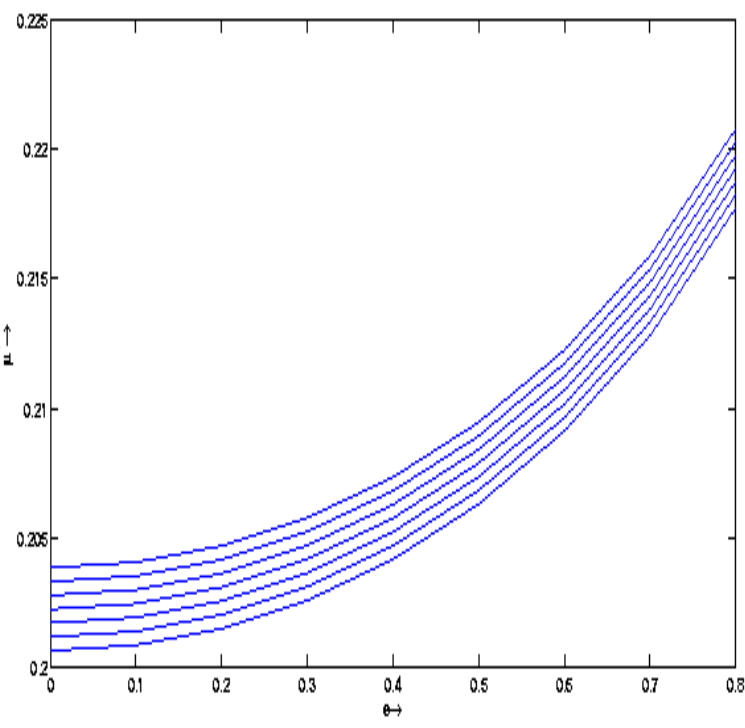

Fig.6: Transition Curves in $\mu^{*}$ and e for $\sigma_{1}=0.003$ to $0.009, \sigma_{2}=0.02$, $\sigma_{1}^{\prime}=0.0005, \sigma_{2}^{\prime}=0.02, \epsilon_{1}^{\prime}=0.0008, \epsilon_{2}^{\prime}=$ and 0.02 .

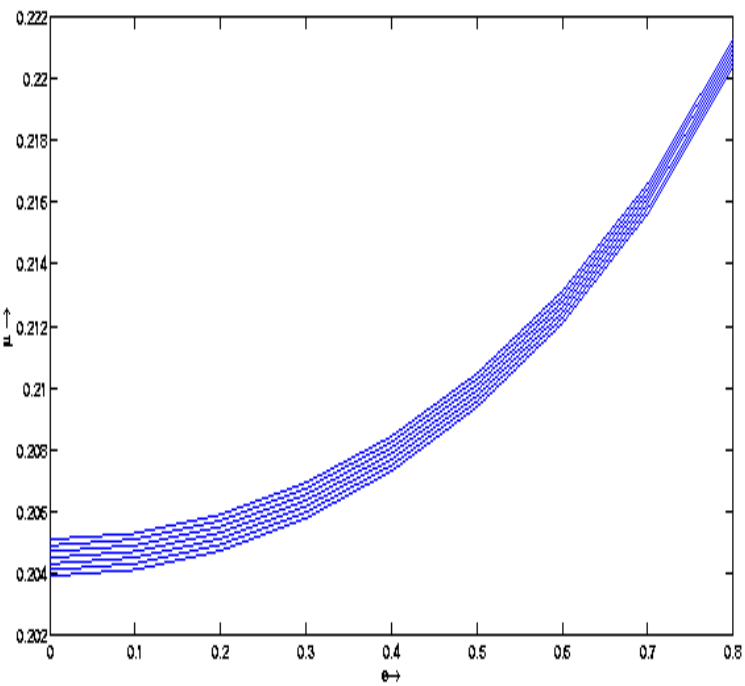

Fig.7:Transition Curves in $\mu^{*}$ and e for $\sigma_{1}=0.005, \sigma_{2}=0.003$ to 0.009 $\sigma_{1}^{\prime}=0.0005, \sigma_{2}^{\prime}=0.02, \epsilon_{1}^{\prime}=0.0008, \epsilon_{2}^{\prime}=$ and 0.02 .

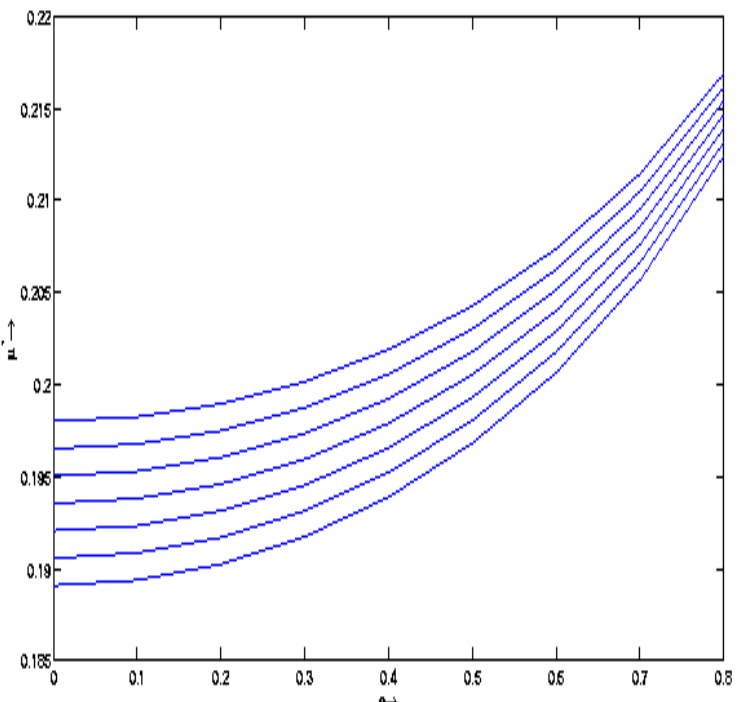

Fig.8: Transition Curves in $\mu^{*}$ and e for $\sigma_{1}=0.005, \sigma_{2}=0.02, \sigma_{1}^{\prime}=0.003$ to $0.009, \sigma_{2}^{\prime}=0.02, \epsilon_{1}^{\prime}=0.0008, \epsilon_{2}^{\prime}=0.02$.

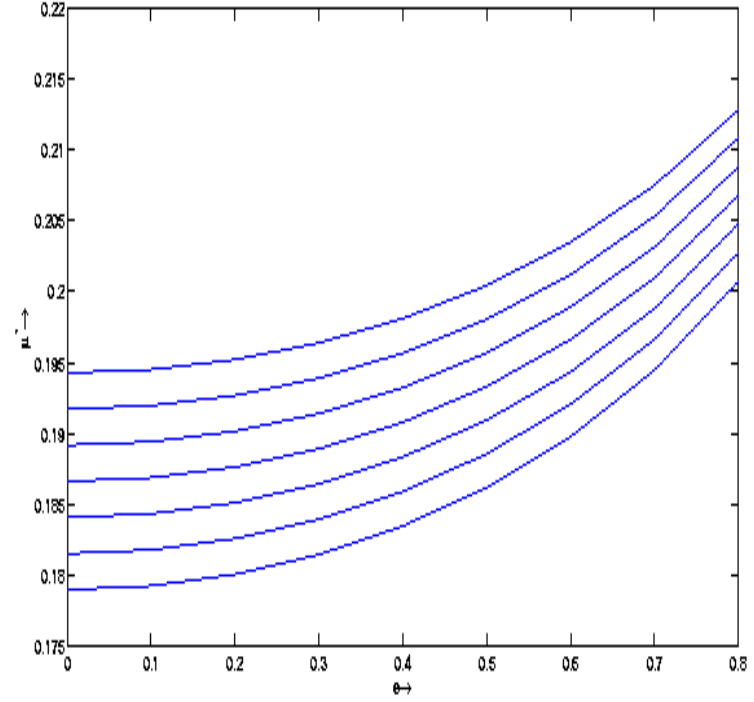

Fig. 9: Transition Curves in $\mu^{*}$ and e for $\sigma_{1}=0.005, \sigma_{2}=0.02, \sigma_{1}^{\prime}=$ $0.0005, \sigma_{2}^{\prime}=0.02, \epsilon_{1}^{\prime}=0.03$ to $0.09, \epsilon_{2}^{\prime}=$ and 0.02 .

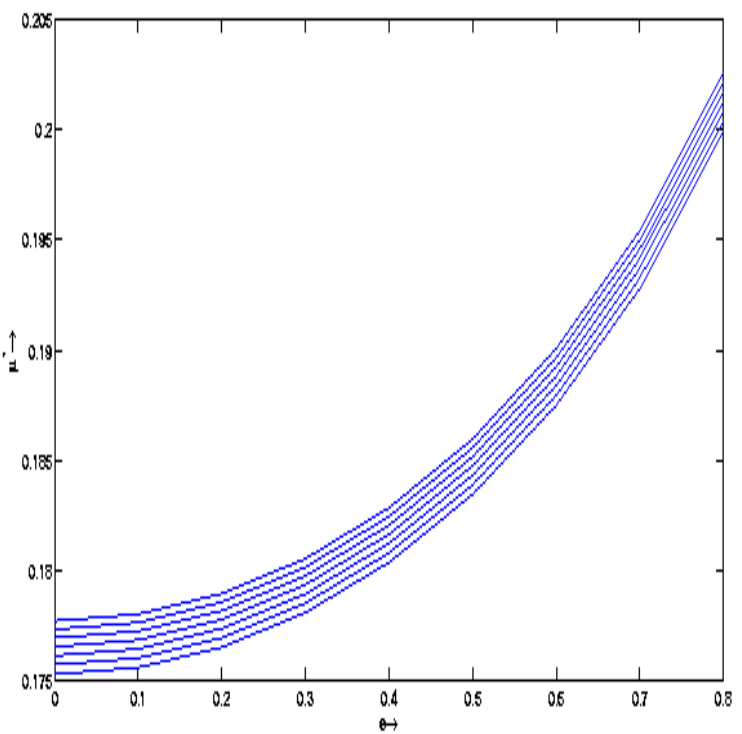

Fig.10:Transition Curves in $\mu^{*}$ and e for $\sigma_{1}=0.005, \sigma_{2}=0.02$, $\sigma_{1}^{\prime}=0.005, \sigma_{2}^{\prime}=0.02, \epsilon_{1}^{\prime}=0.08, \epsilon_{2}^{\prime}=0.03$ to 0.09 .

\section{References}

[1] Ammar MK (2008), The effect of solar radiation pressure on the Lagrangian points in the elliptical restricted three body problem, Astrophysics and Space Science, 313, 393-408. http://dx.doi.org/10.1007/s10509-007-9709-z.

[2] Bennet A (1965), Characteristic exponents of the five equilibrium solutions in the Elliptically Restricted Problem, ICARUS 4, 177-187. http://dx.doi.org/10.1016/0019-1035(65)90060-6.

[3] Danby JMA (1964), Stability of the triangular points in the elliptical restricted problem of three bodies, Astronomical Journal, 69,166-174.

[4] Eilpe A (1985), Ferrer,S. On the equilibrium solution in the circular planar restricted three rigid bodies, Celestial Mechanics, 37, 59. http://dx.doi.org/10.1007/BF01230341.

[5] F A Abd El-Salam (2015), Stability of Triangular equilibrium points in the elliptic restricted three body problem with oblate and triaxial Primaries, AstrophysSpaceSci, http://dx.doi.org/10.1007/s10509-015-2308-5.

[6] Grebnikov E A (1964), On the stability of the Lagrangian triangle solutions of the restricted elliptic three body problem, Soviet Astronomy, 8 , No.3, 567-578.

[7] Grebnikov E A (1986), the methods of Averaging Applications, NAUKA, Moscow Revised.

[8] Gyorgyey J (1985), on the non-linear motions around the elliptical restricted problem of three bodies, Celestial Mechanics and Dynamical Astronomy, 36, No.3, 281-285. http://dx.doi.org/10.1007/BF01230741. 
[9] Ishwar B \&Elipe A (2001), Secular Solutions at Triangular Equilibrium Point in the Generalized Photogravitational Restricted Three Body Problem, Astrophysics and Space Science, 277, No.3, 437-446. http://dx.doi.org/10.1023/A:1012528929233.

[10] Khanna M \&Bhatnagar K B (1998), Existence and Stability of the Libration points in the restricted three body problem when the smaller primary is a triaxial rigid body, Indian Journal of Pure and Applied Mathematics,29(10), 1011-1023.

[11] Kumar V \& Choudhary R K (1990), Non-linear stability of the triangular libration points for the photogravitational elliptic restricted problem of three bodies, Celestial Mechanics and Dynamical Astronomy, 48, No.4, 299-317.http://dx.doi.org/10.1007/BF00049387.

[12] Kumar S \&Ishwar B (2009), Solutions of Generalized Photogravitational Elliptical Restricted Three Body Problems, AIP Conf.Proc. 1146,456.http://dx.doi.org/10.1063/1.3183564.

[13] Markeev A P (1978), Libration points in celestial mechanics and cosmic dynamics, NAUKA Moscow, 312 .

[14] Markellos V V, Perdios E \&Labrapoulou P (1992), linear stability of the triangular equilibrium points in the photogravitational elliptic restricted three body problem, Astrophysics and Space Science, 194, 207-214.http://dx.doi.org/10.1007/BF00643991.

[15] McCusky S W (1963), Introduction to celestial Mechanics, Addison Wesley.

[16]Narayan A \& Kumar C R (2011), Effects of photogravitational and oblateness on the Triangular Lagrangian points in the Elliptical Restricted three body problem, Indian Journal of Pure and Applied Mathematics,68, No.2, 201-224.

[17]Narayan A \&Usha T (2014), Stability of triangular equilibrium points in the elliptic restricted problem of three bodies with radiation and triaxial primaries, Astrophysics and Space Science, 351(1), 135142.http://dx.doi.org/10.1007/s10509-014-1818-x.

[18] Singh, J \&Aishetu U (2012a), Motion in the photogravitational elliptic restricted three body problem under an oblate primary, Astronomical Journal, 143, No.5, 109.http://dx.doi.org/10.1088/0004$6256 / 143 / 5 / 109$.

[19] Singh J \&Aishetu U (2012b), On the stability of triangular points in the elliptic restricted three bodies under the radiating and oblate Primaries, Astrophysics and Space Science ,341, 349358.http://dx.doi.org/10.1007/s10509-012-1109-3.

[20] Subbarao P V \& Sharma R K (1975), A Note on the Stability of the Triangular points of Equilibrium in the Restricted-Three body problem, Astronomy and Astrophysics, 43,381-383.

[21] Szebehely V (1975), Stability of the points of equilibrium in restricted problem, Astronomical Journal, 72,79.http://dx.doi.org/10.1086/110195.

[22] Szebehely V (1967), Theory of Orbits, Academic press, New-York.

[23] Usha T, Narayan A \&Ishwar B (2014), Effects of radiation and triaxiality of primaries on triangular equilibrium points in elliptic restricted three body problem, Astrophysics and Space Science, 349(1), 151164.http://dx.doi.org/10.1007/s10509-013-1655-3.

[24]Zimvoschikova S \&Thkai V N (2004), Instability of libration points and resonance phenomena in the photogravitational elliptical restricted three body problem, Solar system research, 38(2), 155-163. 